\title{
Color Tuning of Face-Selective Neurons in Macaque Inferior Temporal Cortex
}

\author{
DMarianne Duyck, Audrey L. Y. Chang, Tessa J. Gruen, Lawrence Y. Tello, Serena Eastman, \\ Joshua Fuller-Deets, and Bevil R. Conway
}

https://doi.org/10.1523/ENEURO.0395-20.2020

Laboratory of Sensorimotor Research, National Eye Institute, National Institutes of Health, Bethesda, MD 20982-4435

\begin{abstract}
What role does color play in the neural representation of complex shapes? We approached the question by measuring color responses of face-selective neurons, using fMRI-guided microelectrode recording of the middle and anterior face patches of inferior temporal cortex (IT) in rhesus macaques. Face-selective cells responded weakly to pure color (equiluminant) photographs of faces. But many of the cells nonetheless showed a bias for warm colors when assessed using images that preserved the luminance contrast relationships of the original photographs. This bias was also found for non-face-selective neurons. Fourier analysis uncovered two components: the first harmonic, accounting for most of the tuning, was biased toward reddish colors, corresponding to the $\mathrm{L}>\mathrm{M}$ pole of the $\mathrm{L}-\mathrm{M}$ cardinal axis. The second harmonic showed a bias for modulation between blue and yellow colors axis, corresponding to the S-cone axis. To test what role face-selective cells play in behavior, we related the information content of the neural population with the distribution of face colors. The analyses show that face-selective cells are not optimally tuned to discriminate face colors, but are consistent with the idea that face-selective cells contribute selectively to processing the green-red contrast of faces. The research supports the hypothesis that color-specific information related to the discrimination of objects, including faces, is handled by neural circuits that are independent of shape-selective cortex, as captured by the multistage parallel processing framework of IT (Lafer-Sousa and Conway, 2013).
\end{abstract}

Key words: color vision; face perception; inferior temporal cortex; inferotemporal cortex; neurophysiology; social signaling

\section{Significance Statement}

Does the brain encode face-specific color signals, such as those related to health and emotion, through color responses of face-selective neurons? This paper addresses this question by providing the first, to our knowledge, quantitative measurements of the color-tuning of face-selective cells. Face-selective cells are not very responsive to pure color (equiluminant) pictures of faces. But both face-selective and non-face-selective cells are biased for warm colors. Information analysis shows that face-selective cells are not optimally tuned to discriminate face colors but suggests that the cells may contribute to discriminating the reddish component of faces. Alternatively, face-cell color tuning may reflect a broader adaptation of inferior temporal cortex (IT) for the detection of objects, which are, in general, characterized by warmer coloring compared with backgrounds.

Received September 13, 2020; accepted December 12, 2020; First published January 15, 2021.

The authors declare no competing financial interests.
Author contributions: B.R.C. designed research; T.J.G., L.Y.T., S.E., and B. R.C. performed research; M.D., J.F.-D., and B.R.C. contributed unpublished reagents/analytic tools; M.D., A.L.Y.C., and B.R.C. analyzed data; B.R.C. and M.D wrote the paper. 


\section{Introduction}

What role does color play in the neural representation of objects? Some cells in inferior temporal cortex (IT) are shape selective, and many of these cells are also modulated by color (Komatsu and Ideura, 1993; Edwards et al., 2003). Do shape-selective IT cells play a role in discriminating among the typical colors of the shapes to which they are tuned? We take up this question by measuring the color responses of face-selective neurons in fMRIidentified face patches of macaque monkey; we evaluate the possible role of the color tuning by relating the information content at the neural population level with the distribution of face colors (Xiao et al., 2017).

The extent to which face-selective neurons in IT are color-tuned is unsettled. A widespread but not universal assumption is that face cells do not carry color information. The assumption is supported by the observation that luminance contrast is by itself sufficient for face recognition (Kemp et al., 1996; Sinha et al., 2006) and the lack of cross face/color adaptation in psychophysical experiments (Yamashita et al., 2005). Consequently, many studies of face perception use exclusively colorless images (Kanwisher et al., 1997; Ohayon et al., 2012). But while color is not essential for determining face identity, color nonetheless relays important information related to social communication, about health, emotion, and sex (Setchell and Wickings, 2005; Changizi et al., 2006; Waitt et al., 2006; Nestor and Tarr, 2008; Gerald et al., 2009; Leopold and Rhodes, 2010; Webster and MacLeod, 2011; Lefevre et al., 2013; Nakajima et al., 2017; Petersdorf et al., 2017). Moreover, faces viewed under low pressure sodium light (which impairs retinal mechanisms for encoding color) have a paradoxical appearance: they appear green, regardless of race (Hasantash et al., 2019). Such seemingly anti-Bayesian phenomena may arise if neural representations and how they are decoded are optimal with respect to the statistics of the environment (Wei and Stocker, 2015); efficient coding thus predicts a neural population whose tuning curves best discriminate among the most common face colors (Hasantash et al., 2019). One possibility is that this face-relevant color information is encoded by face-selective neurons.

To what extent could face-selective cells discriminate among face colors? Two studies have glanced the question. One found no sensitivity to color among face-selective neurons (Perrett et al., 1982). The other study found that of 22 cells, some showed higher firing rates for

This work was supported by the Intramural Research Program of the National Eye Institute and the National Institute of Mental Health at the National Institutes of Health.

Acknowledgements: We thank the animal care staff at the National Eye Institute, David Leopold, Chris Baker, Arash Afraz, and Rosa Lafer-Sousa, for helpful discussion and Isabelle Rosenthal and Theodros Haile for help with the first stages of the experiment.

Correspondence should be addressed to Bevil Conway at bevil@nih.gov.

https://doi.org/10.1523/ENEURO.0395-20.2020

Copyright @ 2021 Duyck et al.

This is an open-access article distributed under the terms of the Creative Commons Attribution 4.0 International license, which permits unrestricted use, distribution and reproduction in any medium provided that the original work is properly attributed. naturally colored face photographs compared with unnaturally colored photographs (Edwards et al., 2003), suggesting that face-selective neurons carry color signals. Quantitative measurements of color-tuning functions of face-selective neurons have, to our knowledge, not been made, which precludes an answer to the question.

The discrimination potential of a population can be estimated by the Fisher information, which depends on the distribution of tuning peaks, tuning widths, and amplitude modulation across the neural population (Ganguli and Simoncelli, 2010; Fig. 1). If a population activity is to be used to discriminate a given attribute, efficient coding predicts that its Fisher information should reflect the distribution of the attribute in the environment (Wei and Stocker, 2015). If face-selective cells were being used to discriminate face color, then the Fisher information across the population should reflect the natural distribution of face colors. Here, we use fMRI-guided electrode recording of neurons in the middle and anterior face patches of macaque monkeys. We discovered that face-selective cells, as a population, were biased for warm colors. The resulting Fisher Information shows a striking dip that coincides with the peak in the distribution of face colors documented in a large database of measurements of human face colors (Xiao et al., 2017). The results suggest that face-selective neurons are not optimally distributed to enable the discrimination of face colors. We discuss what role color responses of face-selective cells may play in visual processing.

\section{Materials and Methods}

\section{Subjects}

Three male rhesus macaques (Macaca mulatta), weighing 8-10 kg, were implanted with an MRI-compatible plastic (Delrin) chamber and headpost. Surgical implantation protocol has been described previously (Lafer-Sousa and Conway, 2013). Designation of the subjects are M1 (monkey 1), M2 (monkey 2), and M3 (monkey 3). M1 and M3 had chambers over the right hemisphere; M2 had a chamber on the left hemisphere. All procedures were approved by the Animal Care and Use Committee of the National Eye Institute and complied with the regulations of the National Institutes of Health.

\section{Functional imaging targeting of face patches}

The fMRI procedures we use for localizing face patches have been described (Tsao et al., 2006; Lafer-Sousa and Conway, 2013; Rosenthal et al., 2018). Two of the animals (M1, M2) are the same as the animals used in Lafer-Sousa and Conway (2013); the face-patch data and color-tuning data are the same as in the earlier reports. Here, we present an analysis of the fMRI color-tuning data restricted to the face patches of IT. All monkeys were scanned at the Massachusetts General Hospital Martinos Imaging Center in a Siemens 3T Tim Trio scanner. Magnetic resonance images were acquired with a custom-built four-channel magnetic resonance coil system with AC88 gradient insert, which increases the signal-to-noise ratio by allowing very short echo times, providing $1-\mathrm{mm}^{3}$ spatial resolution 

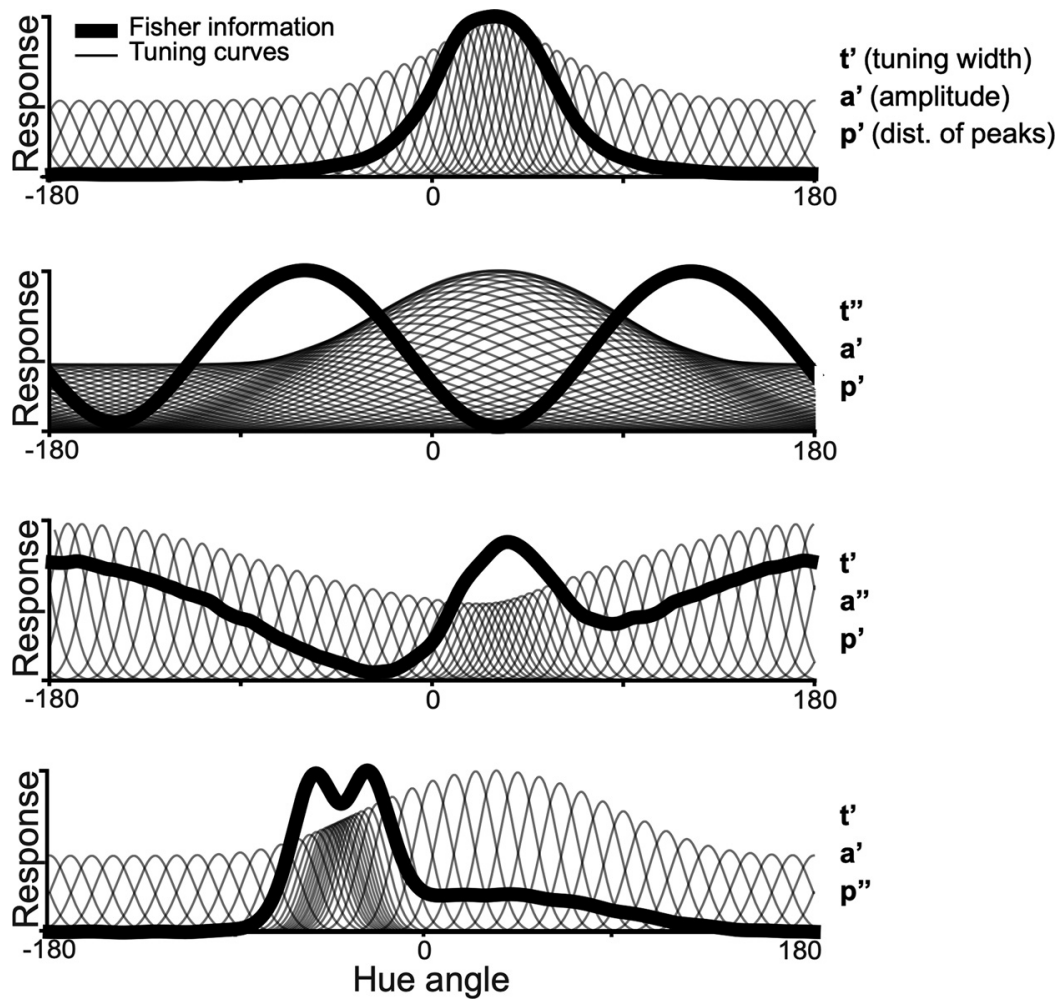

Figure 1. Simulated population of 50 cells responding to a circular variable and corresponding Fisher information. A population with von Mises tuning curves of width t', amplitude a', and distribution of peaks p' yields a Fisher information with one peak. Each of these three parameters can be individually adjusted to create a population with a different Fisher information. Uniformly increasing tuning width (t') while holding a' and p' constant yields a two-peaked Fisher information. Independently adjusting the distribution of tuning curve amplitudes (a") or the distribution of the peaks (p") creates an asymmetric Fisher information.

and good coverage of the temporal lobe. We used standard echo planar imaging (repetition time $=2 \mathrm{~s}, 96 \times$ $96 \times 50$ matrix, $1 \mathrm{~mm}^{3}$ voxels; echo time $=13 \mathrm{~ms}$ ). Monkeys were seated in a sphinx position in a custom-made chair placed inside the bore of the scanner, and they received a juice reward for maintaining fixation on a spot presented at the center of the screen at the end of the bore. An infrared eye-tracker (ISCAN) was used to monitor eye movements, and animals were only rewarded by juice for maintaining their gaze within $\sim 1^{\circ}$ of the central fixation target. Magnetic resonance signal contrast was enhanced using a microparticular iron oxide agent, MION (Feraheme, $8-10 \mathrm{mg} / \mathrm{kg}$ of body weight, diluted in saline, AMAG Pharmaceuticals), injected intravenously into the femoral vein just before scanning.

Visual stimuli were displayed on a screen subtending 41 by 31 degrees of visual angle (dva), at $49 \mathrm{~cm}$ in front of the animal using a JVC-DLA projector (1024 $\times 768$ pixels). The subset of presented stimuli used here to localize face patches consisted in achromatic square photographs of faces and body parts presented centrally on a neutral gray screen $\left(\sim 25 \mathrm{~cd} / \mathrm{m}^{-2}\right)$ and occupying $6^{\circ}$. They were shown in 16 32-s blocks (16 repetition times per block, repetition time $=2 \mathrm{~s}$, two images per repetition) presented in one run sequence. The images were matched in average luminance to the neutral gray, maintaining roughly constant average luminance $\left(\sim 25 \mathrm{~cd} / \mathrm{m}^{-2}\right)$ throughout the stimulus sequence. For faces stimuli we used: 16 unique images front-facing of unfamiliar faces (eight human, eight monkey) repeated twice within a block. The bodies/ body parts block comprised 32 unique images of monkey and human bodies (no heads/faces) and body parts. Face patches were localized by contrasting responses to faces versus responses to body parts. A total of 18 runs were used to localize face patches in $\mathrm{M} 1$ and $\mathrm{M} 2$, and 16 runs were used to localize face patches in M3.

\section{Physiologic recordings}

A plastic grid was fitted to the inside of the recording chamber to enable us to reproducibly target regions within the brain, following details reported previously (Conway et al., 2007). We used sharp epoxy-coated tungsten electrodes $(\mathrm{FHC})$, propelled using a hydraulic manual advancer (Narishige). Voltage traces were digitized and saved with a Plexon MAP system (Plexon Inc.). Spike waveforms were sorted offline with the Plexon Offline Sorter, and single units were defined on the basis of waveform and interspike interval.

Recordings were performed in a light-controlled room, with the animals seated in sphinx position. Animals were acclimatized to head restraint to minimize head movement during recordings. Animals maintained fixation on a spot on a monitor $57 \mathrm{~cm}$ away; the monitor was a CRT Barco subtending 40 by $30 \mathrm{dva}$, operating at $85 \mathrm{~Hz}$ and at a resolution of $1024 \times 768$ pixels. Eye position was 
monitored throughout the experiments using and infrared eye-tracker (ISCAN). The monitor was color calibrated using a PR-655 SpectraScan Spectroradiometer (Photograph Research Inc.); we achieved 14-bit resolution for each phosphor channel using Bits ++ (Cambridge Research Systems).

\section{Stimuli}

Screening stimuli consisted in 10 grayscale exemplars of each of the 14 following categories: animals, buildings, human faces (front view), monkey faces (front view), human faces (3/4 view), monkey faces (3/4 view), fruits, furniture, monkey bodies (no face), human bodies (no face), places, technology objects, indoor places, natural scenes, vehicles (see examples Fig. 2). All stimuli were presented on a static luminance white noise background of 7.5 by $7.5^{\circ}$ of visual angle.

Stimuli for the color-tuning experiments were exemplars of faces of unfamiliar humans and monkeys, front or $3 / 4$ view (16 exemplar of frontal view and 8 of $3 / 4$ view for each species), fruits known to the monkey (16 exemplars) and bodies/body parts (without head) of unfamiliar humans and monkeys (eight exemplars for each subcategory), yielding a total of 96 stimuli. For both sets of experiments using colored stimuli (main condition in which the colored stimuli preserved the luminance contrast of the original achromatic images, and the equiluminant condition), we defined 16 target hues equally spaced along the CIELUV color space (values provided in Table 1). For the main condition (Fig. 3), each pixel value of the original achromatic image was remapped to the most saturated target color of the same luminance value within the monitor gamut. For the equiluminant condition, each pixel value of the original image was remapped to a pixel on the equiluminant plane, of the same hue as the target but different saturation based on the pixel luminance. Determination of equiluminance was Judd-Vos corrected for the underestimation of the contribution of S-cones to the standard luminosity function (Vos, 1978).

Note that one way of making false-colored images that has been used in some studies involves the digital equivalent of superimposing a color filter over a black and white picture. In these images, the white of the original image is replaced with a relatively saturated color. Although easy to generate, these false-colored images are luminance compressed compared with the original image: the black in the colored version remains the same luminance as the original, but the white is now a lower luminance than the white in the original. Moreover, it is possible that the estimation of luminance for a given color is inaccurate (for discussion, see Conway, 2009). Such inaccuracies would introduce variability in the luminance contrast among the different colored images generated using the color-filter method. For example, when an achromatic image is falsely colored by applying a color filter, such that the white in an original image is replaced with a given equiluminant color, the resulting set of differently colored, photometrically equiluminant, versions of the image may have different luminance-contrast ranges that systematically vary by hue: the red, yellow, and green images may have a higher luminance range than the blue and purple images, because the contribution of S-cones to the luminosity function is underestimated. Thus, despite being ostensibly equiluminant, the brightest blue in the blue image may be lower luminance than the brightest red in the red image, yet the black will be the same luminance in both images. Variation in the responses to differently colored images created in this way cannot be interpreted as color tuning because they may reflect variable sensitivity to the range of luminance contrasts in the set of differently colored images. The method used presently mitigates the possible impact of chromatic aberration and variability in macular pigmentation, and gives rise to images that are more naturalistic: the images not only retain the luminance contrast of the original images but also appear differently colored, rather than achromatic but viewed through a colored filter (see Golz and MacLeod, 2002).

\section{Procedure}

One experimental session started by targeting a microelectrode to a face patch, recording a single unit (selected online based on waveform), mapping the receptive field by hand, and choosing the visual-field location that gave the highest response to the screening stimuli. Then we recorded the neural activity while presenting the battery of screening stimuli. If a cell appeared face selective, we then recorded responses to the colored stimuli presented in random order. During both screening and main experiment stimuli were presented for $200 \mathrm{~ms}$ followed by a 200-ms blank (gray background). The animals were rewarded by juice for maintaining their gaze within $\sim 1^{\circ}$ of the central fixation dot for a specific duration (that duration usually started at $3 \mathrm{~s}$ but was decreased during the experiment to adapt to the animal motivational state). Once a cell was found recording took place until the animal stopped working thus yielding a variable number of trials per session, ranging from 369 to 15,355 (Interquartile range $(I Q R)=[2945,6755])$. Across all sessions the number of repetitions for a given stimulus by hue combination ranged between 0 and 15 . Equiluminant stimuli were presented in a subset of sessions.

\section{Data analysis}

\section{Response window}

The response of each neuron was quantified within a response window defined using the average response to all stimuli, in 10-ms bins. The baseline firing rate of each cell was defined as the average response from $50 \mathrm{~ms}$ before stimulus onset to $10 \mathrm{~ms}$ after stimulus onset. The response window for each cell was determined as one continuous time period initiated when, within two consecutive time bins, the neural response increased above 2.5 SDs above the baseline firing rate and terminated either when the response dropped below 2.5 SDs of the baseline firing rate in two consecutive bins or after $200 \mathrm{~ms}$ following the start of the response window (the shorter of the two options was used). Cells were only included in the analysis if 
the response window was initiated between 50 and 250 ms after stimulus onset, and if the neural response was excitatory (i.e., cells showing suppressive responses to stimulus onset were not included).

\section{Face selectivity}

The present analysis focused on the color tuning of face-selective neurons. Face selectivity was assessed using the following index:

$$
F S I=\frac{R_{\text {face }}-\operatorname{mean}\left(R_{\text {bodies }}, R_{\text {fruits }}\right)}{\left|R_{\text {face }}\right|+\mid \text { mean }\left(R_{\text {bodies }}, R_{\text {fruits }}\right) \mid},
$$

where $R$ is the average response to stimulus, computed as the difference between the firing rate during the response window and the firing rate during background. Face-selectivity index (FSI) values range from -1 to +1 , with values above 0 indicating a higher response to faces compared with bodies and fruits. All analyses, with one exception, were restricted to neurons that showed an FSI $\geq 1 / 3$, corresponding to a response to faces at least twice that of the response to other non-face stimuli. The exception was the last analysis (see Fig. 12), in which we examined the relationship between hue preference and face selectivity. For that last analysis we included an additional 61 cells (ML: 49 and AL: 12) that were recorded by targeting the same face patches but had an FSI below 1/3. The total number of recorded cells was thus 234 (74\% of the targeted cells had an $\mathrm{FSI}$ of at least 1/3).

\section{Color tuning}

To ensure that the results are not tainted by any cells that were not face selective, we focus on the responses to color of those 173 cells that showed a FSI $\geq 1 / 3$. An analysis of the color responses of the entire population of recorded neurons, which included some cells with low FSI, is shown in Figure 12. In the color-tuning analyses we pooled responses across the different face stimuli for each hue. Across all cells, pooling over stimuli, the number of trials per hue ranged from 10 to $634(\mathrm{IQR}=[119$, 277]).

\section{Significance}

We determined for each cell whether there were significant variations in net firing rate across the 16 hues by computing the coefficient of variation (the ratio of the SD across hues to the mean) of the data recorded for the neuron compared with the distribution of coefficients of variation obtained by 1000 permutations of hue labels. We considered color modulation to be significant when the $p$ value was below $\alpha=0.05$.

\section{Description: vector sum}

Color responses were also quantified by determining the vector sum of the color response. This analysis is enabled because hues are circularly distributed: we can consider the neuron's response to a hue as a vector whose direction is the hue angle, and the vector norm is the strength of the response within the response window compared with baseline. We normalized the vector norms among hues so that the total sum over hues was one. The strength of the hue preference is estimated by the direction and norm of the vector sum. Equation 1 describes the normalized vector computed for each hue; we then sum these vectors using Equation 2:

$$
\begin{gathered}
\overrightarrow{\boldsymbol{v}}_{\text {hue }}=\frac{\left(F R_{\text {hue }}-\min \left(F R_{\text {hues }}\right)\right) /\left(\max \left(F R_{\text {hues }}\right)-\min \left(F R_{\text {hues }}\right)\right)}{\sum_{\text {hue }=1}^{16}\left[\left(F R_{\text {hue }}-\min \left(F R_{\text {hues }}\right)\right) /\left(\max \left(F R_{\text {hues }}\right)-\min \left(F R_{\text {hues }}\right)\right)\right]} \\
*\left[\begin{array}{c}
\cos \theta_{\text {hue }} \\
\sin \theta_{\text {hue }}
\end{array}\right] \\
\overrightarrow{\boldsymbol{v}}=\sum_{\text {hue }=1}^{16} \overrightarrow{\boldsymbol{v}}_{\text {hue }} .
\end{gathered}
$$

The preferred hue direction of the cell is the angle of $\vec{v}$. The strength of the hue preference is the norm of $\overrightarrow{\boldsymbol{v}}$, and can take values ranging from 0 (no hue preference) to 1 . The norm of the vector sum therefore reflects the narrowness of the color tuning.

\section{Description: Fourier analysis}

Color responses can be analyzed using Fourier analysis (Krauskopf et al., 1982; Stoughton et al., 2012) that identifies the set of sine waves (frequency, phase angle, and amplitude) that capture the shape of the color-tuning function. We extracted for each cell the normalized amplitude and phase angle of the first eight harmonics; most of the power was captured by the first two harmonics. The first harmonic has a single peak when plotted in polar coordinates of color space (i.e., a vector pointing to one color), the second harmonic identifies an axis in these coordinates (i.e., the poles of the axis identify an opponent color pair). Confidence interval of the mean was obtained by resampling cells with replacement and computing the angular mean 1000 times (for the second harmonic, for all cells we used the peak between $0^{\circ}$ and $180^{\circ}$ ).

\section{Correlation between fMRI and electrophysiology}

We correlated the single cell activity for each of the 16 hues for all single cells of all three monkeys separately for each face patch, to the average percent signal change to these 16 hues obtained by interpolating from the signal change to the 12 hues used in the fMRl experiment [facepatches were identified over the two hemispheres of M1 and M2 of the current study, details of stimuli and region of interest (ROI) definition can be found in Rosenthal et al., 2018]. Note that to make the Bold response and neurons' firing rate more comparable, we averaged the net firing rate over the entire window from stimulus onset to the onset of the next stimulus $(400 \mathrm{~ms})$. The firing rate is thus lower than when selecting a response window tailored to each cell.

\section{Population information}

To compute the population of face-selective cells' Fisher information, we fitted each cell's net average firing rate response to the 16 hues by a von Mises function of the form $r(\theta)=a+b \exp \left(\kappa\left[\cos \left(\theta-\theta_{\text {pref }}\right)\right]\right)$, with $\kappa>0$, $a>0$ and $b \geq 0$ (median mean squared error across all cells of 0.38 spikes/s). The population Fisher information is given by: 
Table 1: Coordinates of the target hues and adaptation point in CIE xyY, CIE LUV, and cone contrast spaces

\begin{tabular}{|c|c|c|c|c|c|c|c|}
\hline & \multicolumn{3}{|c|}{ CIE xyY } & \multicolumn{2}{|c|}{ CIE Luv } & \multicolumn{2}{|c|}{ DKL } \\
\hline & $x$ & y & $\mathrm{Y}$ & u' & v' & LM & $\mathrm{S}$ \\
\hline Hue $0^{\circ}$ & 0.3473 & 0.3183 & 56.5 & 0.2268 & 0.4677 & 0.0979 & 0.0547 \\
\hline Hue $45^{\circ}$ & 0.3607 & 0.3675 & 56.5 & 0.2157 & 0.4945 & 0.0760 & 0.3347 \\
\hline Hue $67.5^{\circ}$ & 0.3536 & 0.3884 & 56.5 & 0.2034 & 0.5027 & 0.0483 & 0.4026 \\
\hline Hue $90^{\circ}$ & 0.3370 & 0.4010 & 56.5 & 0.1889 & 0.5056 & 0.0145 & 0.4123 \\
\hline Hue $157.5^{\circ}$ & 0.2659 & 0.3704 & 56.5 & 0.1538 & 0.4822 & -0.0819 & 0.1164 \\
\hline Hue $180^{\circ}$ & 0.2505 & 0.3450 & 56.5 & 0.1510 & 0.4677 & -0.0979 & -0.0547 \\
\hline Hue $202.5^{\circ}$ & 0.2441 & 0.3196 & 56.5 & 0.1538 & 0.4532 & -0.0995 & -0.2282 \\
\hline Hue $225^{\circ}$ & 0.2465 & 0.2980 & 56.5 & 0.1621 & 0.4409 & -0.0852 & -0.3754 \\
\hline Hue $247.5^{\circ}$ & 0.2563 & 0.2826 & 56.5 & 0.1744 & 0.4326 & -0.0562 & -0.4678 \\
\hline Hue $270^{\circ}$ & 0.2717 & 0.2747 & 56.5 & 0.1889 & 0.4298 & -0.0171 & -0.4851 \\
\hline
\end{tabular}

$$
I_{F}(\theta)=\left(\frac{\partial \ln (p[\mathbf{r} \mid \theta])}{\partial \theta}\right)^{2}
$$

Assuming independent Poisson noise, we can derive that:

$$
I_{F}(\theta)=\sum_{n=1}^{173} \frac{\left(f_{n}^{\prime}(\theta)\right)^{2}}{f_{n}(\theta)}
$$

where $f_{n}$ represents the tuning function for cell $n$.

For visualization, we also present the population information smoothed with a Savitsky-Golay filter (window of $50^{\circ}$, first order polynomial). We also computed the $95 \%$ confidence intervals of the Fisher information using nonparametric bootstrapping with 1000 iterations.

Finally, we performed the same analysis with the original 16 CIELUV hue angles projected along the two chromatic axes: $180-0^{\circ}$ corresponding to greenish to reddish hues, and $270-90^{\circ}$ corresponding to bluish to yellowish hues.

\section{Distribution of natural face color}

Xiao et al. (2017) measured the spectra of skin on the cheek, forehead, back of hand and inner arm of 960 participants of four ethnicities (White, Chinese, Kurdish, and Thai) under D65 illuminant, and reported the mean and SD of the values for each body part and each ethnicity in CIELAB color space. We averaged the forehead and cheek $\mathrm{L}, \mathrm{a}^{*}$ and $\mathrm{b}^{*}$ means and SDs (Table 2 from Xiao et al., 2017 ). We then computed a weighted average across all ethnicities for both mean and SD (using Table 1 from Xiao et al., 2017). Using standard conversion matrices from CIELAB to $X Y Z$ and $X Y Z$ to CIELUV, we obtained an estimate of the mean and $S D$ of the distribution of face color in CIELUV hue angle, represented as a von Mises distribution in Figure 11.

\section{Results}

Functional magnetic resonance imaging was used to identify regions of IT that were more responsive to faces than to bodies and fruits, a standard contrast used to identify face patches (Tsao et al., 2006; Lafer-Sousa and Conway, 2013). We targeted microelectrodes to the ML face patch in two monkeys (M1 and M2) and the AL face patch in three monkeys (M1, M2, and M3; Fig. 2A). To screen for face-selective neurons, we measured the responses of each cell to a battery of grayscale images of 14 categories (Fig. 2B). Face-selective neurons, such as the two examples in Figure $2 B$, were defined as those that showed at least a 2-fold greater response to faces than bodies and fruits. This selection criterion yielded 102 single units in $\mathrm{ML}$ and 71 single units in AL. Face-selective cells in $\mathrm{AL}$ had a higher FSI than cells in $\mathrm{ML}\left(M d n_{\mathrm{ML}}=\right.$ 0.60, $M d n_{\mathrm{AL}}=0.92$, Mann-Whitney-Wilcoxon rank-sum test $U=1842, p<0.001$; Fig. $2 C$ ); cells in $A L$ and $M L$ had similar firing rates $\left(M d n_{\mathrm{ML}}=6.38, M d n_{\mathrm{AL}}=6.28, U=3671\right.$, $p>0.88$ ). All face-selective cells showed a significant main effect of face image (repeated measures ANOVA on a cell-by-cell basis; responses were the average firing rate during the response window to each face images; analysis restricted to the 102 neurons that were tested with at least three presentations of each face image; all neurons were $p<0.05)$. The preferred face was more

Table 2: Number of cells, median latency, and duration (in ms) of the response window used for the main analysis, by mon-

\begin{tabular}{|c|c|c|c|c|c|c|c|c|c|c|c|c|}
\hline & \multicolumn{3}{|c|}{ M1 } & \multicolumn{3}{|c|}{ M2 } & \multicolumn{3}{|c|}{ M3 } & \multicolumn{3}{|c|}{ Total } \\
\hline & \#Cells & Latency & Duration & \#Cells & Latency & Duration & \#Cells & Latency & Duration & \#Cells & Latency & Duration \\
\hline$\overline{\mathrm{ML}}$ & 45 & 125 & 180 & 57 & 75 & 150 & - & - & - & 102 & 95 & 175 \\
\hline$A L$ & 7 & 135 & 160 & 15 & 95 & 130 & 49 & 75 & 60 & 71 & 85 & 110 \\
\hline
\end{tabular}
key and by face patch 
A

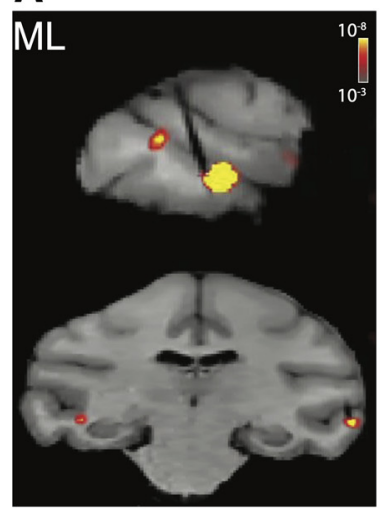

B

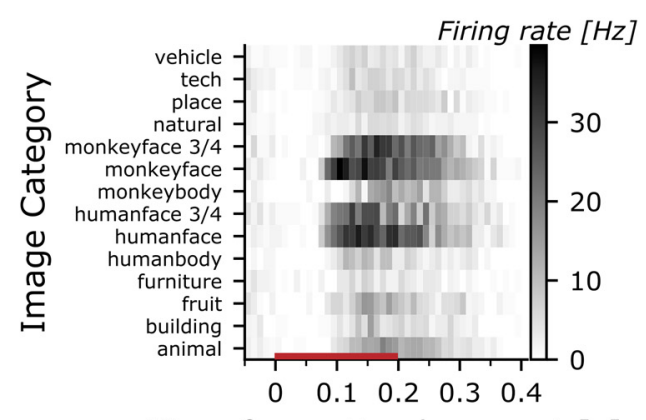

Time from stimulus onset [s]
C
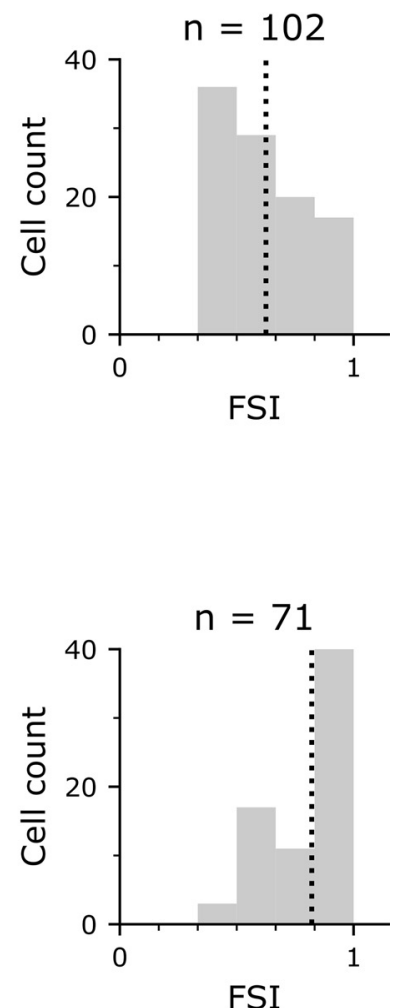

FSI
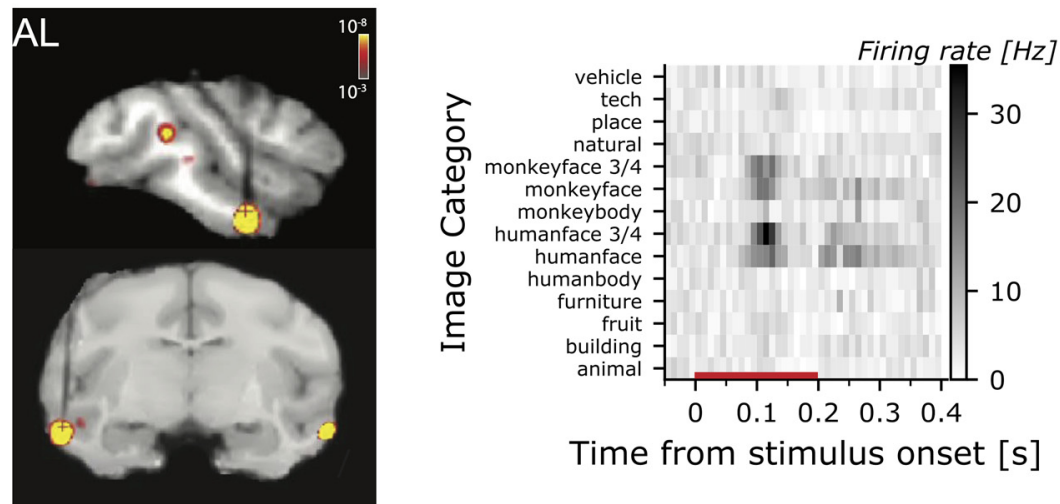

Figure 2. FMRI-guided microelectrode recording of face-selective cells in macaque IT. $\boldsymbol{A}$, MR Images with recording microelectrodes; superimposed is shown the fMRI contrast maps of faces>bodies uncover the face patches (ML-M2, top; AL-M3, bottom). Electrodes appear black in the MRI images, and target the face patches. B, PSTH for an example cell in ML (top) and AL (bottom) showing the responses to achromatic images used to identify face cells. The red line along the $x$-axis shows the stimulus duration. $\boldsymbol{C}$, Histogram showing the FSI for the population of face cells in ML (top) and AL (bottom); the dashed vertical line shows the mean $\mathrm{FSI}$. All cells with an FSI $>1 / 3$ were included in the analysis.

likely a monkey face ( $70 \%$ of the cells), and more likely a $3 / 4$ view than a front view (64\% of the cells). Across all cells, the preferred stimulus triggered a firing rate with a median 2.3 times higher than when using the average we are using for all analyses (IQR $=[1.7,3.0])$.

We next measured responses to color for the face-selective neurons. Color responses were obtained using monochromatic versions of photographs of faces, bodies, and fruits (Fig. 3A); the colored stimuli evenly sampled CIELUV color space (Fig. 3B, top panel). We chose to define the stimuli in CIELUV color space because this space captures the representation of color within the V4 Complex (Bohon et al., 2016), which provides input into IT (Kravitz et al., 2013). The chromaticity of the stimuli can be transformed into cone-opponent "DKL" space, which reflects the cone-opponent cardinal mechanisms evident in the lateral geniculate nucleus (Derrington et al., 1984; Sun et al., 2006; Fig. 3B, bottom panel). Evaluating color responses in DKL space is useful because it has a physiological basis; throughout the paper, the CIE colors corresponding to the poles of the cone-opponent axes ( $L>M$, $\mathrm{M}>\mathrm{L}, \mathrm{S}+$, and $\mathrm{S}-)$ are provided to facilitate this evaluation. To create a given image in a target color, we replaced each pixel in the original gray-scale image with the target hue of the same luminance value as the pixel. Thus, the false-colored images maintained the luminance contrast of the original image.

Figure 4 shows the responses of a representative sample of six face-selective neurons to the colored images of faces, bodies, and fruit; cells 1-3 were recorded in face patch ML; cells 4-6 were recorded in face patch $A L$. The top panels show the average responses to images of faces, bodies, and fruits. As predicted given the screening criterion, responses were always substantially larger to faces than to the other stimulus categories, with FSIs ranging from 0.44 to $\sim 1$. For each cell, we defined a time period for quantification of the responses (Fig. $4 A, B$, blue bars). We used a single continuous time period for all cells whose duration was tailored to each cell. The response of cells showed complex temporal dynamics. For example, cell \#4 showed two peaks (at 105 and $225 \mathrm{~ms}$ ) and the intervening firing rate dipped back to baseline, in cells such as this one, the time period for quantification only included the initial peak. Using multiple time periods for some cells such as cell \#4 did not change the main conclusions (data not shown). The median latencies and the 

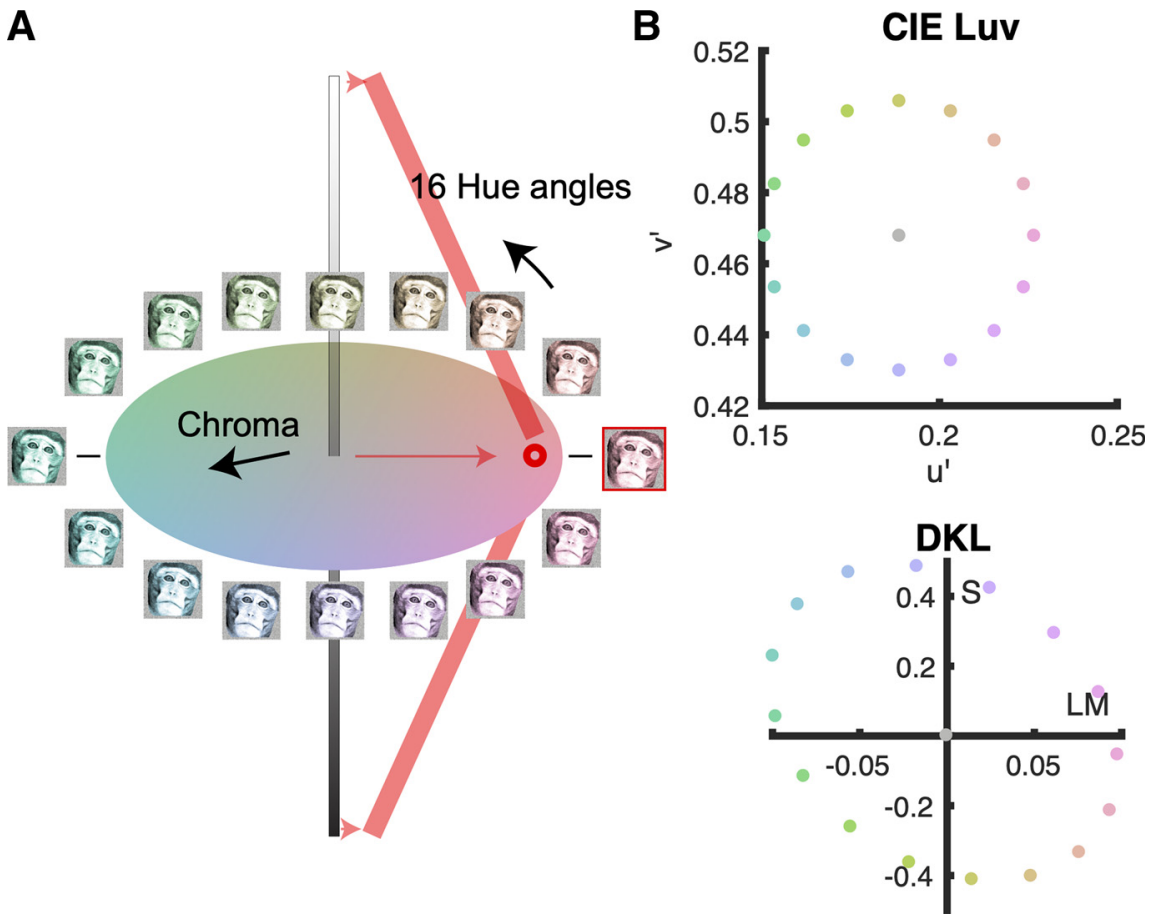

Figure 3. Stimuli used to measure the color responses of face-selective cells in macaque IT. $\boldsymbol{A}$, Color space illustrating the procedure for generating the colored images. Each colored image was generated by replacing the pixels in the original achromatic image with a given hue that preserved the luminance of original pixel. $\boldsymbol{B}$, Sixteen colored versions were generated; the chromaticity of the most saturated pixel in each version is shown in CIELUV ( $u^{*}, v^{*}$; top panel) and cone-opponent color space (DKL, bottom panel).

duration of the response time periods across the population of cells are reported in Table 2. Cells in ML showed a shorter latency than cells in AL within each monkey. But the variability in latencies for cells in $M L$ or $A L$ across monkeys was greater than the difference in latency between $M L$ and $A L$ within any monkey.

Figure $4 B$, top panels, shows poststimulus time histograms (PSTHs) of the responses to the colored faces, averaging across the four different face categories we used (monkey and human faces, frontal faces and 3/4view faces; see Fig. 2B). The orientation of the PSTH shows time on the $y$-axis and image color on the $x$-axis. The stimulus onset is at $0 \mathrm{~s}$, and darker gray corresponds to higher firing rate. The responses of the neurons are delayed by a latency reflecting the time for visual signals to be processed by the retina and relayed through the visual-processing hierarchy to IT. The cells in Figure 4 were representative of the population: three of the cells were modulated by the color of the stimulus (cells \#1, \#3, \#6), reflected in the average response over the response window (black trace below the PSTH; for significance calculation, see Materials and Methods). Among the population of face-selective neurons, $\sim 25 \%$ were significantly modulated by color (23/102 cells in ML; $21 / 71$ cells in AL). Cells \#2, \#4, and \#5 were not modulated significantly by color. Figure $4 B$, red traces, shows the best-fitting sine wave following Fourier analysis of the color responses, described below.

Note that the firing rates shown in Figure $4 B$, bottom panels, are averages over the response window and so are lower than the peak firing rates shown in Figure $4 A$.
Although many cells were modulated by color, the variance in firing rate caused by changes in color was modest. For example, the firing rate in cell \#3 varied between 18 and 22 spikes/s above background, which corresponds to $\pm 10 \%$ of the average stimulus-driven response. Across the population, the variation in firing rate because of hue was $\pm 24 \%$ of the average stimulus-driven response. Approximately $76 \%$ of the stimulus-driven response can be therefore attributed to the luminance contrast of the images.

Figure $4 C$ plots the cells' responses as a function of color angle; the norm of the vector sum is shown as the bolded black line, and varies between 0 and $1(0=$ equal net firing rate for all hues identical, i.e., no color tuning; values for each cell are shown in black text). To further quantify the results, we determined the best-fitting sine wave of the color-tuning response for each cell (Fig. 4B, red lines). Many cells $(71 / 173)$ were best fit by the first harmonic (a single cycle), which shows that these cells have a single preferred color (see cells \#3, \#6; Fig. 4), but some cells were best fit by the second harmonic (21/173), indicative of a preference for a color axis in color space, rather than a single-color direction (cell \#1; Fig. 4). The amplitude and phase angle of the best fitting harmonic is shown in red in Figure 4C. The color preferences assessed by the norm of the vector sum and the normalized amplitude of the first harmonic were highly correlated (Pearson $r=0.93, p<0.001$ ). Among the 44 cells showing significant color modulation, the power of both the first and the second harmonic was higher than the noise level estimated as the power to the eighth harmonic (Fig. 5, red 
ML
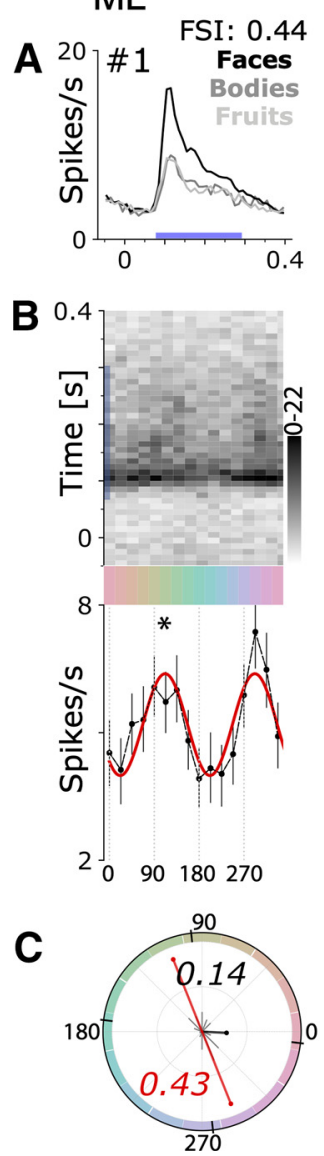
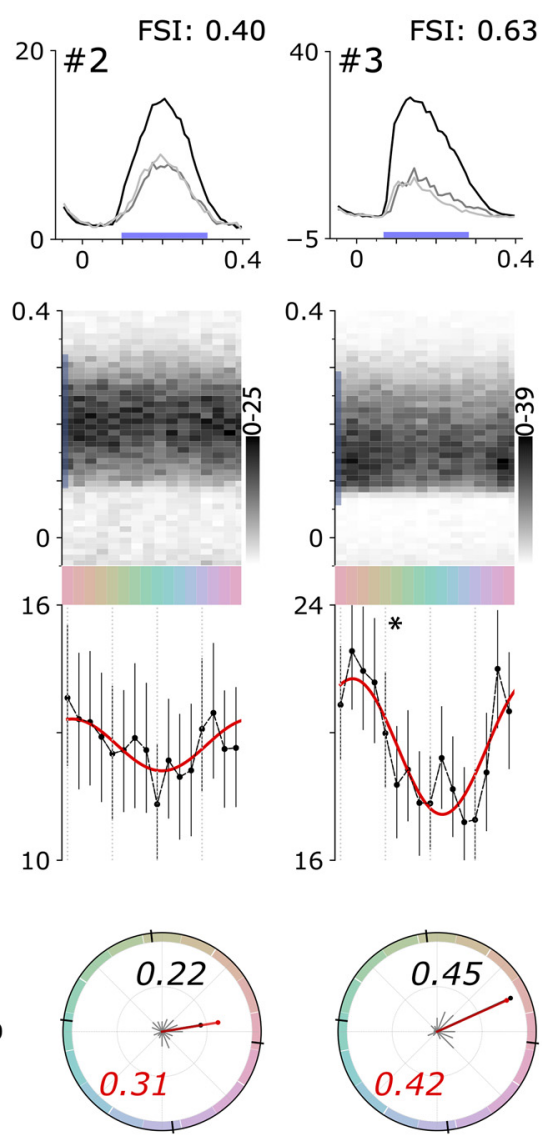
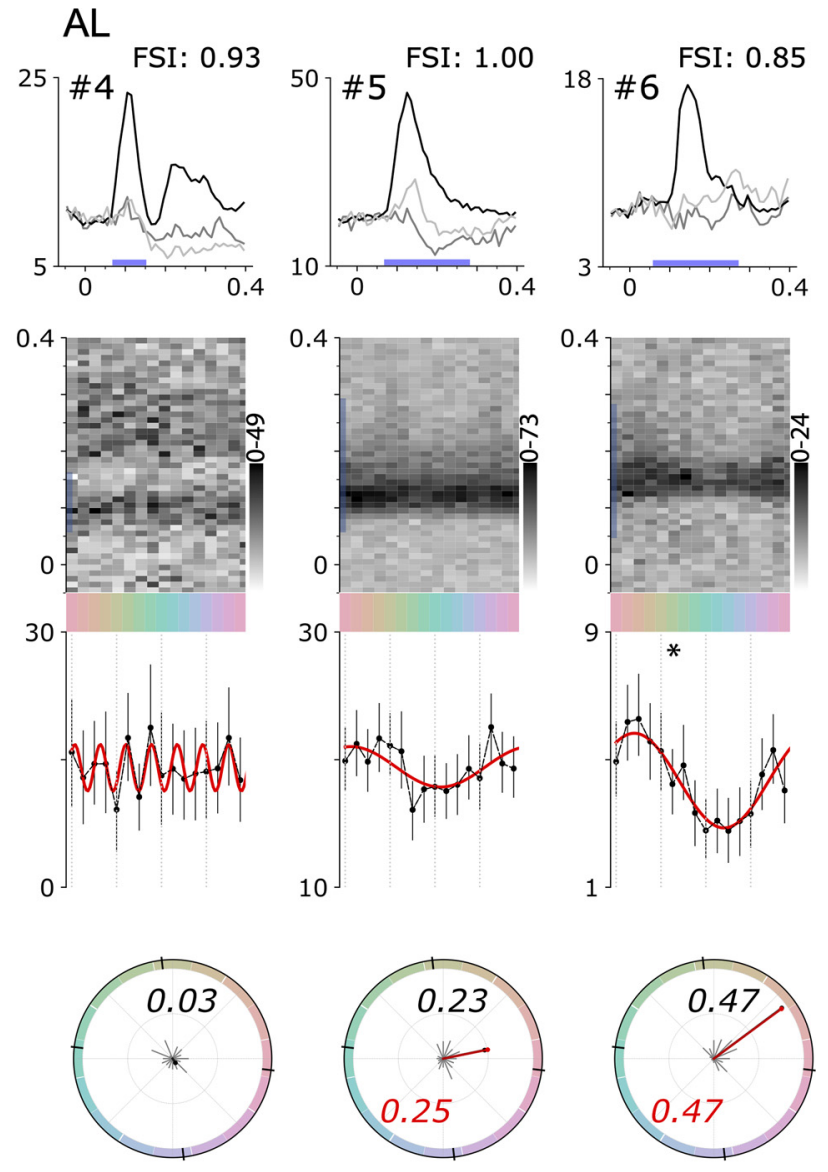

Figure 4. Responses to color of six face-selective cells in macaque IT (two cells for each monkey M1: \#2, 3; M2: \#1, 5; M3: \#4, 6). $\boldsymbol{A}$, Average responses to images of faces, bodies, and fruits. The time period during which the responses were quantified in subsequent analyses is shown by the blue bar along the $x$-axis. $\boldsymbol{B}$, PSTH (top panels) showing the average responses to colored images of faces; blue bar along the time axis as in panel $\boldsymbol{A}$. Average response to face images of each color (bottom), quantified during the time period indicated by the blue bar in $\boldsymbol{A}, \boldsymbol{B}$. Error bars show 95\% confidence intervals; the red line shows the best fitting sine wave, and an asterisk is provided if the color tuning for the neuron was significant (see Methods). $\boldsymbol{C}$, Polar plot showing normalized responses to all hues; the sum of the responses to the 16 colors is normalized to equal 1 . The bold black text states the norm of the vector sum. The red line shows the normalized amplitude and phase of the best fitting sine wave for neurons whose best fit was a first or second harmonic; the red text states the value of the normalized amplitude of the best-fitting sine. The small black lines on the edges of the circle show the cardinal axes of the cone-opponent color space.

lines); $37 / 44$ cells showed highest power to the first harmonic; $5 / 44$ showed highest power to the second harmonic; $1 /$ 44, to the third; and $1 / 44$ to the fourth. We found no evidence that the color selectivity of the cells depended on the cells' face preference: for each neuron, we computed the color selectivity (as the normalized amplitude of the first Fourier component) for each face image. We then rank-ordered the face images by descending average firing rate and ran a repeated measures ANOVA with the ranks as independent variables. There was no-significant main effect of the rank on color selectivity $(p>0.13)$.

Figure 4 provides evidence that some face-selective cells were sensitive to color. Among the population, was there a consistent color preference? Figure 6 shows the color responses of all the face-selective cells rank-ordered by the significance of the color tuning, with the most significantly color-tuned cells at the top. Each row shows data from one cell. The gray level shows the normalized response to the given color (the sum of the values across colors for a given cell is 1 ). Many of the most significantly color-tuned neurons in both $\mathrm{ML}$ and $\mathrm{AL}$ preferred warm colors, as evident by the dark regions on the upper left and right of the panels in Figure 6. But there were some exceptions. For example, cells represented by rows 6 and 7 in the ML panel and rows 1 and 2 in the AL panel of Figure 6 showed a preference for greenish colors.

Figure 7 quantifies the color responses of the population of single cells using Fourier analysis. Figure $7 A$, left panel, shows a polar histogram of the peak color direction for cells with maximum power to the first harmonic; significantly color-tuned cells are shown in dark gray. These results confirm the population bias toward the red pole of the $0-180^{\circ}$ chromatic axis, corresponding to the $L>M$ pole of the L-M cone-opponent axis. This bias is also evident when analyzing the color direction of the best-fitting first harmonic for all cells in the population (including those that did not have maximum power in the first harmonic; Fig. 7B, left panel). In contrast, cells with maximum 


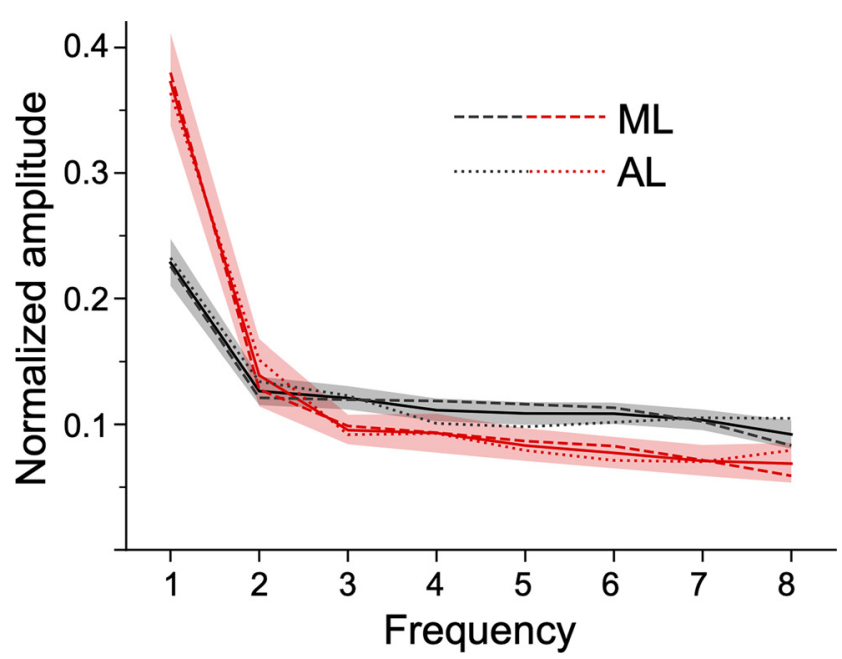

Figure 5. Power spectrum of the Fourier analysis of the colortuning responses of face-selective neurons in macaque IT. Average normalized amplitude of each harmonic component for the population of 173 cells (in black) and for significantly colortuned cells (in red). Surrounding shaded areas show 95\% confidence intervals. Dashed and dotted lines represent the averages for, respectively, $M L$ and $A L$ and solid lines the average across both face patches.

power to the second harmonic showed a phase angle biased for the $90-270^{\circ}$ axis, corresponding to modulation along the $\mathrm{S}$-cone axis (Fig. 7A, right panel). And this bias was also evident when analyzing the best fitting second harmonic for all cells in the population (including those
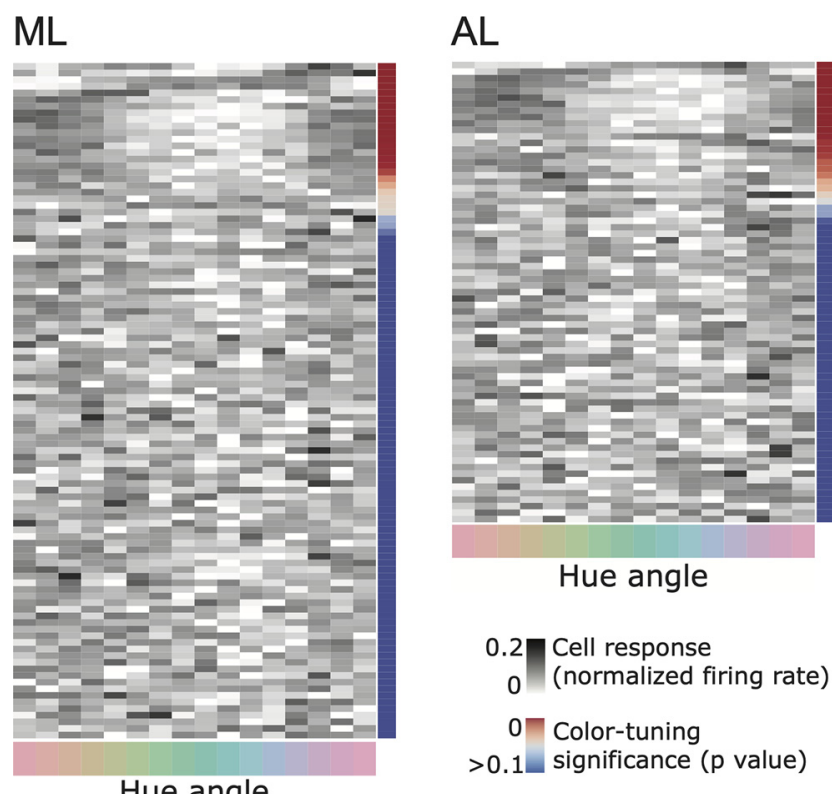

Figure 6. Responses to face images in each of 16 colors, for each cell in the ML face patch (left) and the AL face patch (right). Each row shows data for one cell. Cells are ordered from the top by descending color selectivity ( $p$ value indicated by the color scale). The plot shows normalized responses: the sum of the responses to the 16 colors for each row adds up to one (darker gray indicates relatively stronger responses). that did not have maximum power in the second harmonic; Fig. 7B, right panel). The pattern of results shown in Figure 7 was evident when analyzing data for each animal separately (Fig. 7C).

How does color tuning relate to color selectivity? If color tuning reflects a computational operation of the circuit one might predict that within the population more color-selective cells will have more consistent color tuning. Figure 8 quantifies the polar direction of the norm of the vector average (i.e., the peak color preference; $y$-axis), color selectivity ( $x$-axis), significance of color tuning (symbol gray value), and number of stimulus repeats obtained (symbol size). The data points to the right of the plot converge on $0^{\circ}$ (the $\mathrm{L}>\mathrm{M}$ pole of the $\mathrm{L}-\mathrm{M}$ axis), consistent with the prediction. Significantly color-tuned neurons, defined by the $p<0.05$ threshold had a mean preferred hue angle that did not differ from insignificantly color-tuned neurons (Watson-Williams test, $F_{(1,171)}=0.07, p=0.80$ ) but had a significantly lower variance (marginal distribution, Wallraff test, $\chi^{2}=11.59, p<0.001$; Fig. 8). This effect cannot be attributed to variance in the amount of data collected for different neurons. Indeed, splitting the population into two groups, above and below the median number of trials collected per cell, yielded a similar variance in the peak color for two groups (Wallraff test, $\chi^{2}=$ $1.10, p=0.29$ ).

The face-selective neurons responded strongly to all the colored stimuli, even those of suboptimal color (see Fig. 4). We attribute the strong color-independent responses to the luminance contrast of the stimuli (regardless of the color, the stimuli preserved the luminance contrast of the original images). We can directly dissect the role of color and luminance contrast on the cell responses by using equiluminant colored stimuli (Fig. 9A). These stimuli were created by replacing the range of gray values in the original images with colors of a constant gray value but different saturation: higher luminance gray values were replaced with more saturated color. Responses to these equiluminant stimuli were substantially lower than responses to the colored stimuli that preserved luminance contrast $\left(M d n_{\text {Iso }}=0.99, M d n_{\text {Main }}=7.64\right.$, Wilcoxon rank-sum test $U=4643, p<0.001$; Fig. 9B). These results show that pure color is not sufficient to strongly drive face-selective cells. Because there is no accepted metric for relating color contrast and luminance contrast (Shevell and Kingdom, 2008), it is often difficult to compare responses to equiluminant stimuli with responses to luminance contrast stimuli. In the present study, this difficulty is mitigated for several reasons. First, the maximum color contrast of the equiluminant stimuli was the highest that the gamut of the display could produce. If color were a sufficient drive of the neural activity of face-selective neurons, the equiluminant stimuli we used should elicit strong responses. Second, using stimuli of comparable color and luminance contrast, other neurons in the visual system show clear preferences for the color stimuli (in V1: Conway, 2001; Johnson et al., 2004; Horwitz and Hass, 2012; in V4: Conway et al., 2007; Bohon et al., 2016; in IT: Komatsu and Ideura, 1993; Lafer-Sousa and Conway, 2013), confirming that these stimuli are capable of eliciting strong responses when neurons are responsive to color. 

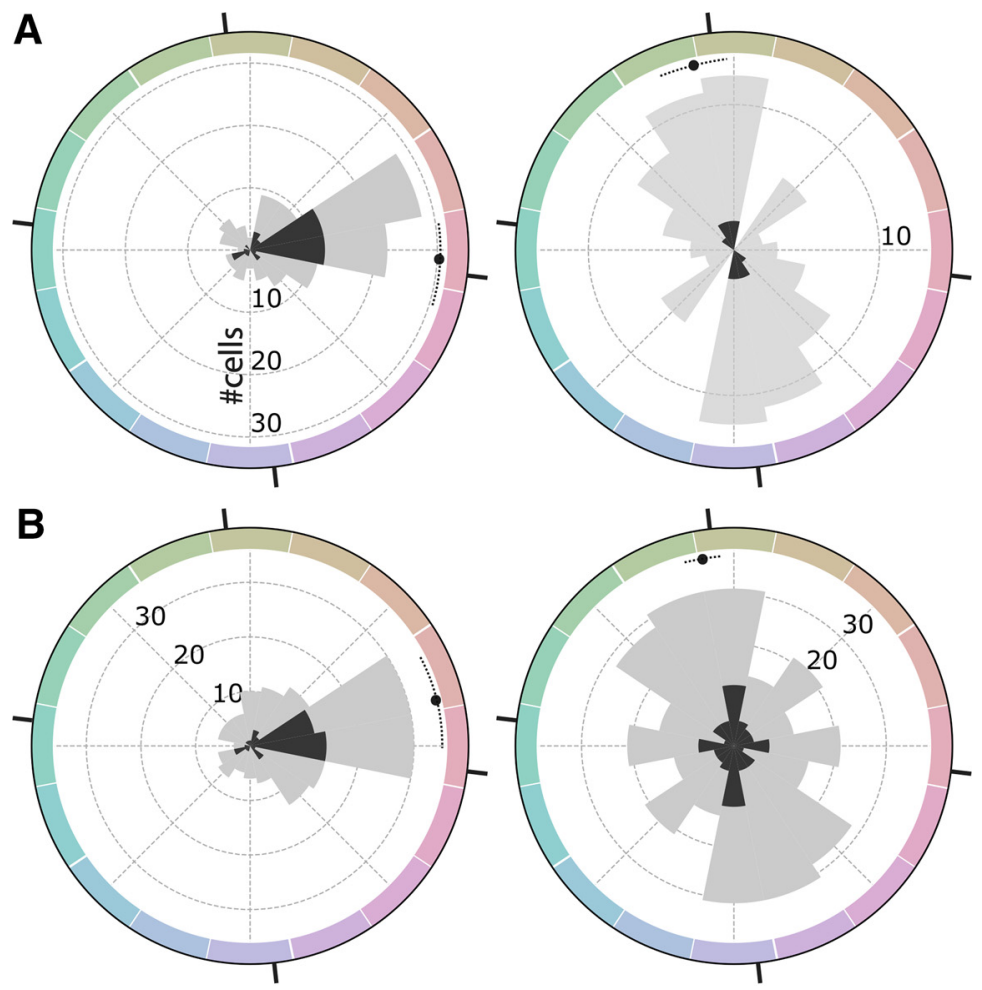

C
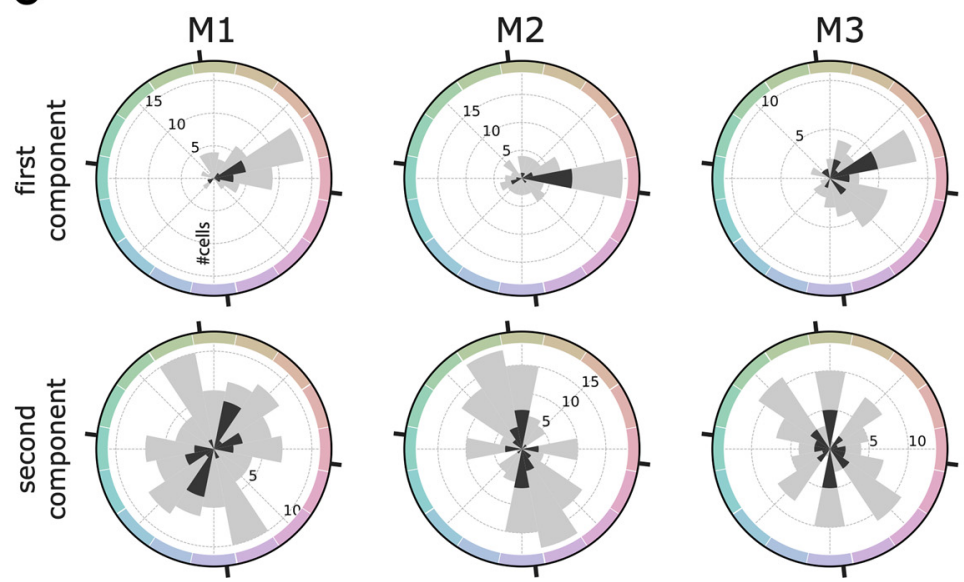

Figure 7. Fourier analysis of the color responses of face-selective cells in macaque IT. $\boldsymbol{A}$, left panel, Distribution of the phase angle of the first harmonic component for cells with higher amplitude in the first harmonic than the second harmonic (126/173 cells; mean: $\left.-4.40^{\circ} \mathrm{Cl}=[-17.0,+8.5]\right)$. Right panel, Phase angle of the second harmonic component for cells with higher amplitude in the second harmonic compared with the first harmonic $\left(47 / 173\right.$ cells; mean: $\left.+102.7^{\circ} \mathrm{Cl}=[+94.5,+111.1]\right)$. $\boldsymbol{B}$, Distribution of the phase angle for the whole population (173 cells) for the first harmonic (left panel; mean $+13.3^{\circ} \mathrm{Cl}=[-0.1,+27.9]$ ) and the second harmonic (right panel; mean: $+99.6^{\circ} \mathrm{Cl}=[+94.2,+105.3]$ ). The color space is CIELUV; black tick marks are provided for the cardinal axes of the cone-opponent DKL color space (these are offset from the axes of CIELUV by $6.7^{\circ}$ ). $\boldsymbol{C}$, Distribution of the phase angles of the two first Fourier components across all cells for each monkey plotted separately.

We previously measured the color responses across IT using fMRI (Lafer-Sousa and Conway, 2013; Rosenthal et al., 2018). To directly compare the results of the fMRI with the cell data, we quantified the neural responses within a 400-ms time window starting at the stimulus onset. This time window encompasses the 200-ms duration of the stimulus and the 200-ms interstimulus gray period. Figure $10 \mathrm{~A}$ shows the average response for the population, in $\mathrm{ML}$ (solid line) and AL (dotted line). The plot shows significant differences among the responses to the colors (non- parametric Friedman test, $\chi^{2}=210.69, p<0.001$ ) and the responses in $\mathrm{ML}$ are highly correlated with those in $\mathrm{AL}$ (Pearson $r=0.89, p<0.001$ ). Figure $10 B$ shows the average response across all face-selective cells to face images in each of the 16 colors. This plot underscores two main conclusions. First, responses to all colored images were strong, which we attribute to the fact that all the colored exemplars preserved the luminance contrast of the original achromatic images, the luminance contrast is a main determinant of face-selective responses (Ohayon et 


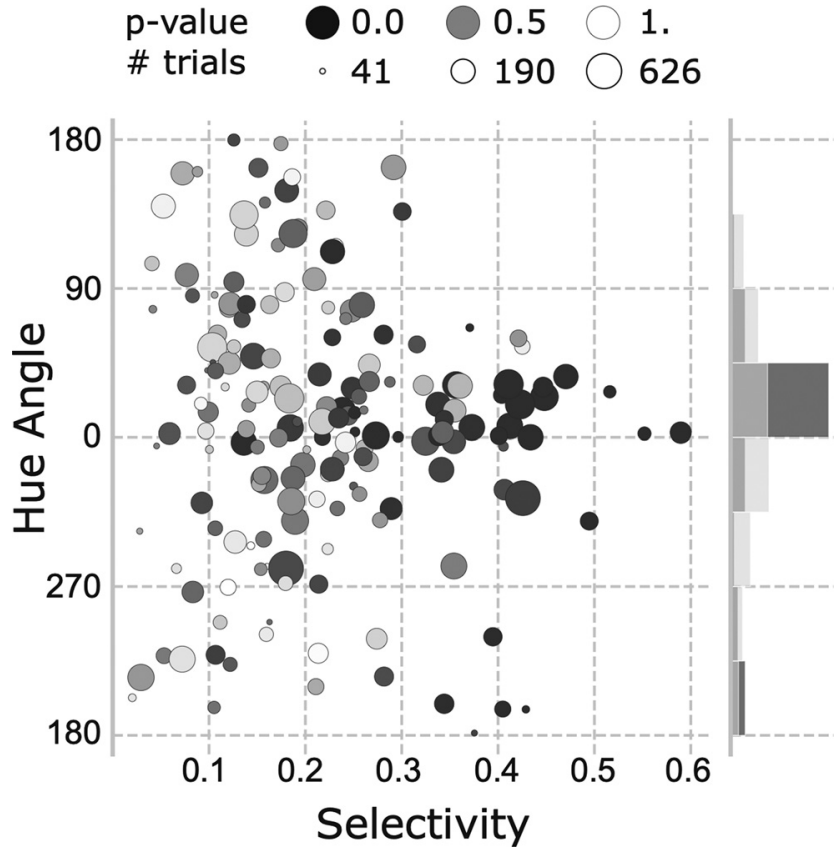

Figure 8. Quantification of the color responses of face-selective cells. Preferred hue angle (direction of the average vector) is plotted as a function of the strength of the color preference (norm of the average vector). Each face-selective cell is represented by a dot. The symbol size corresponds to the median number of stimulus presentations per hue. The gray value of the symbols reflects the significance of the color modulation $p$ value). The marginal distribution shows the normalized distribution of the preferred hue for significantly (dark gray) and nonsignificantly (light gray) color-tuned cells.

al., 2012). And second, among the colored stimuli, responses to the $\mathrm{L}>\mathrm{M}$ stimuli (appearing pinkish) were higher than response to the $\mathrm{M}<\mathrm{L}$ stimuli (appearing greenish). The color biases of the population of face-selective cells were strongly correlated with the color biases of the face patches measured with fMRI (ML: Pearson $r=0.67, p=0.005$, power $=0.84 ;$ AL: Pearson $r=0.61$ $p=0.01$, power $=0.75$; Fig. $10 \mathrm{C}$ ).

The data presented above quantify the color-tuning properties of face cells. If the color responses of face-selective cells reflect a role these cells play in discriminating face colors, we predicted that the Fisher information of the population would correspond to the distribution of face colors. The color statistics of face skin are available in a large database of calibrated measurements derived from multiple ethnicities (Xiao et al., 2017). We assume that the color statistics of bare macaque face skin shows a comparable bias to that found across humans (and we assume that neural measurements in macaque monkeys extend to the human case). Figure $11 \mathrm{C}$ shows the Fisher information computed for the neural data as a function of hue angle using von Mises function to describe the cells' response (Fig. 11A). Superimposed on the panels is the distribution of face colors (dashed curves). The peak of the distribution of face colors does not correspond to maxima in the Fisher information; to the contrary, the likely colors of faces correspond to a dip in the Fisher
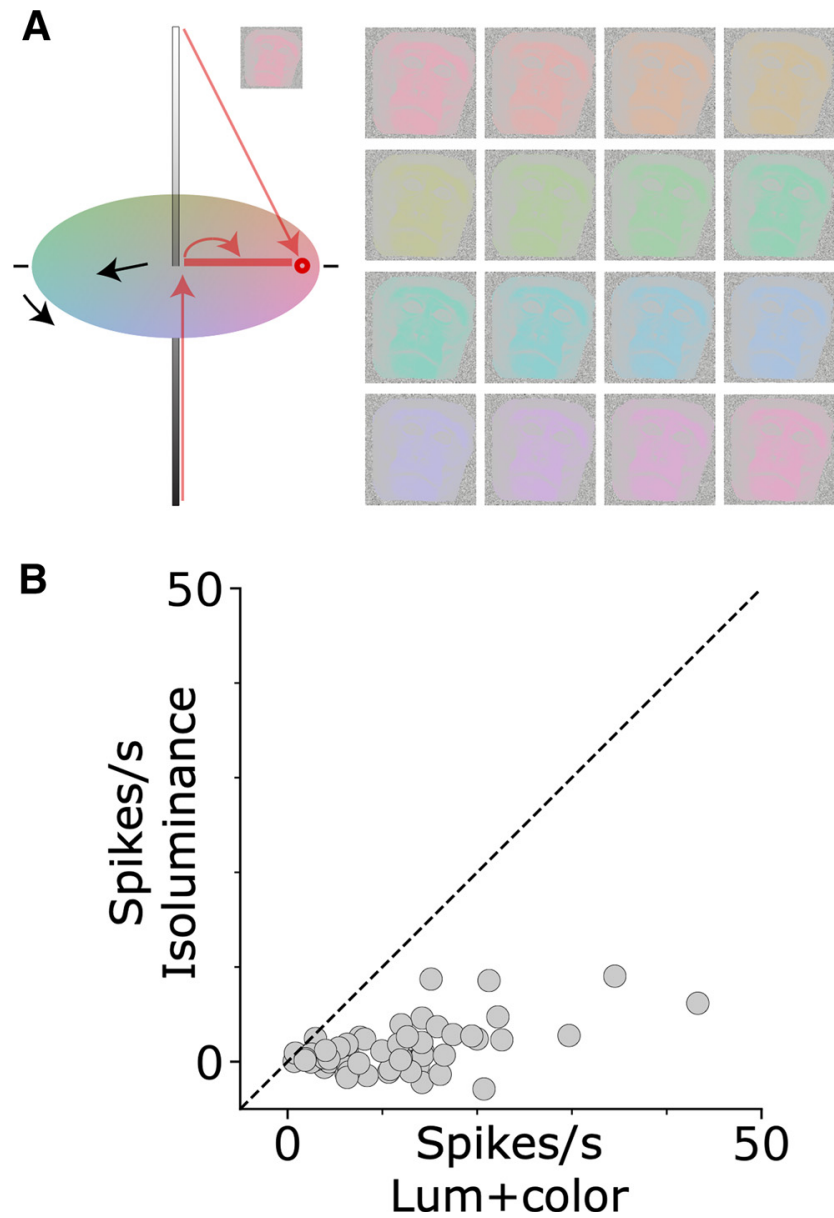

Figure 9. Response to equiluminant stimuli. $\boldsymbol{A}$, Illustration of the construction of an $\mathrm{L}>\mathrm{M}$ colored image. The range of gray values in the original image were replaced with colors defined by a vector along an equiluminant plane in the color space; white pixels of the original image were rendered in a saturated hue, black pixels were rendered in gray, and gray pixels were rendered in a hue of intermediate saturation. $\boldsymbol{B}$, Firing rate above background of a population of face-selective neurons to equiluminant stimuli ( $x$-axis) versus luminance-preserved colored stimuli $(y$-axis; $N=71$ cells, $M L=35, A L=36$ ), each dot represents one cell.

information, which implies that the population is poor at discriminating the colors of faces. We did the same analysis projecting the original data along the $0-180^{\circ}$ axis in CIELUV space, which approximates the L-M axis (Fig. $11 B, C$, middle panels), to evaluate whether face cells contain more information about the color component of faces that is most relevant for dynamic social signaling (the red pole of the green-red axis; Hasantash et al., 2019). In this analysis, the Fisher information peaks for reddish colors, consistent with the idea that face cells are color-tuned in a way that can contribute to the discrimination of $L>M$ values. For comparison, Figure $11 B, C$, right panels, shows the analysis for data along the vertical axis in CIE color space, which approximates the S-cone axis. Faceselective cells did not show selective tuning along the S-cone axis; the Fisher information analysis implies that the cells do not carry as much information along the Scone axis as they do along the L-M axis. 


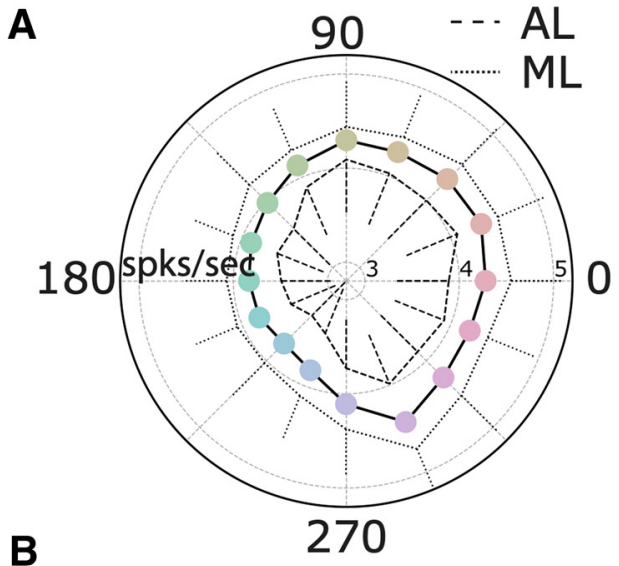

B
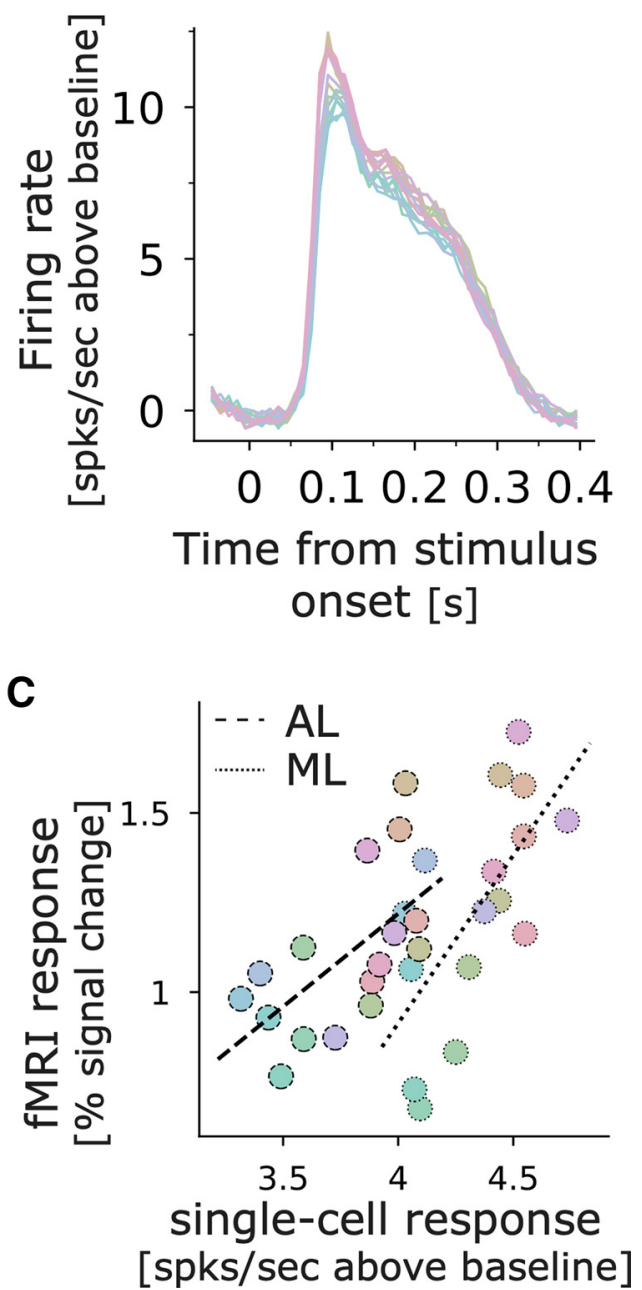

Figure 10. Comparison of color tuning measured using microelectrode recording of single cells in face patches and fMRI. $\boldsymbol{A}$, Average above-background firing rate computed over a 400-ms time window that begins with the stimulus onset (peak responses away from 0 can be accounted for by summing the first two harmonics of the response). $\boldsymbol{B}$, Average above-background response for all face-selective cells $(N=173)$ to face images in 16 colors (the color of the traces corresponds to the colors of the images, see Fig. 3). $\boldsymbol{C}$, Correlation between average response across the population of single units and fMRI color tuning assessed in the face patches of monkeys M1 and M2 (see Materials and Methods).

\section{Discussion}

The population of face-selective neurons showed broad color tuning with a bias for reddish colors. The Fisher information, which reflects how well the neural population can discriminate among colors, shows a dip which coincides with the peak in the distribution of face colors measured across human ethnicities (Xiao et al., 2017). This pattern of results implies that face-selective cells in macaque IT are not optimally tuned to discriminate the colors of human faces. It is conceivable that face-selective neurons in macaque IT are optimally tuned to discriminate macaque face colors, although this would require a substantial difference in the colors of macaque faces compared with human faces, which is unlikely since the primary determinants of face coloring (oxygenated hemoglobin and melanin) are the same in both species. It is also conceivable that the color responses of the neurons may be stronger if color was manipulated in spatial patterns on the face to reflect the spatial distribution of natural color changes over the face. Thus, the color responses we report provide a lower bound. The color tuning curves were measured using images that preserve the normal luminance contrast relationships of face photographs. In a second series of experiments, we found that face-selective cells were not very responsive to pure color (equiluminant) images of faces, which underscores the importance of luminance contrast for face selectivity (Ohayon et al., 2012), and provides single-unit evidence supporting the fMRI observation that face patches respond more strongly to luminance contrast compared with equiluminant color (Lafer-Sousa and Conway, 2013). Taken together with prior work, the research supports the idea that color-specific information related to the discrimination of face colors is likely handled by neural circuits that are independent of face patches. This interpretation is consistent with the multistage parallel processing framework of IT, in which face-biased regions are largely non-overlapping with color-biased regions (Conway, 2018).

We related the neurophysiology results to behavior using an information framework. Optimal neural coding suggests that there should be a good match between neural tuning and the statistics of those parts of the environment that are relevant (Simoncelli and Olshausen, 2001; Ganguli and Simoncelli, 2010). Faces occupy a distinct gamut in color space (Crichton et al., 2012; Chauhan et al., 2015; Xiao et al., 2017). If face-selective cells participate in discriminating among the colors of faces, the Fisher information of the population of neural responses should correspond to the distribution of face colors. The neurophysiological results refute this prediction. Most of the significantly color-tuned face-selective neurons cells were best described as having broad tuning, with a single peak in the color-tuning function. On average, the colortuning peaks across cells were to warm $(L>M)$ colors (Fig. 7), corresponding to the typical colors of faces. The Fisher information curve is bimodal and the color-discrimination potential of face-selective neurons is therefore worse for face colors compared with greens and purples (Fig. 11, peaks at 84 and -66 hue angle).

These results suggest that some other population of neurons is responsible for discriminating the colors of 

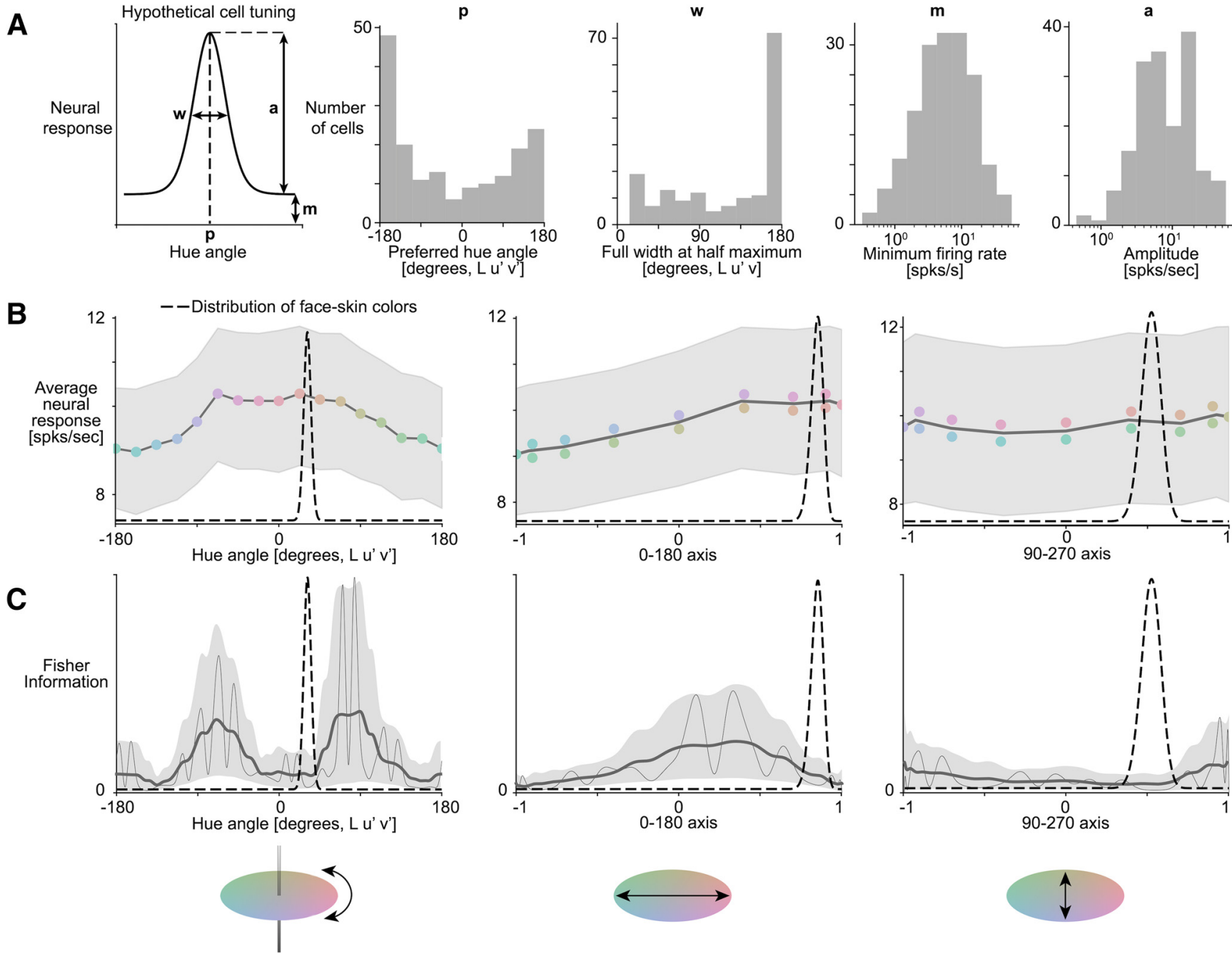

Figure 11. Analysis of the Information represented in the population. A, Parameters of the von Mises fits over the 173 face-selective cells used to compute the population information. $\boldsymbol{B}$, Average net firing rate across the population. $\boldsymbol{C}$, Population Fisher information (thin line), smoothed Fisher information (thick line), and 95\% confidence intervals. On both panels, the dashed line represents the distribution of natural face skin color, and the $y$-axis limits for Fisher information is kept constant across all three analyses. The left column corresponds to the analysis performed in CIELUV space, the middle one to the analysis projected along the greener to redder chromatic axis (positive values indicating redder), and the last one to the analysis projected along the bluer to yellower chromatic axis (positive values indicating yellower).

faces. Functional MRI response patterns in both macaque monkeys and humans show a multistage organizational scheme governed by a repeated eccentricity template, in which color-biased tissue is sandwiched between faceselective tissue (foveal biased) and place-selective tissue (peripheral biased) in parallel streams along the length of the ventral visual pathway (Lafer-Sousa and Conway, 2013; Lafer-Sousa et al., 2016; Conway, 2018). This organization provides the possibility that color-specific information about objects, including faces, could be extracted by neural circuits besides the face patches. But we note that within the face-selective population we studied, some cells were color-tuned with peak tuning away from reddish colors; these cells were, curiously, the most color selective in the population (Fig. 6, top rows). These neurons may represent a distinct category of face-selective cells that could, conceivably, be optimally tuned to discriminate the colors of faces.
What role, if any, does the color tuning of face cells play in visual processing? We consider three possibilities. First, the information content with regards to color was not zero, so the cells could discriminate face colors, but non-optimally. Second, the color component of faces to which humans are most sensitive, regardless of race, is the aspect that varies in response to changes in emotion and health, which is encoded selectively along the L-M chromatic axis (Hasantash et al., 2019). Could the color tuning of face-selective neurons optimally discriminate just this component? Consistent with this possibility, we found that the average tuning and the Fisher information increased for stimuli with larger $L>M$ values. The pattern of results is similar to the ramp-tuning functions of face-selective neurons for other stimulus features (Freiwald et al., 2009). Thus, the selectivity we observed implies that the extent of L-M color contrast in a face is a relevant feature encoded by face-selective neurons. Finally, we wonder whether the color tuning may serve to 


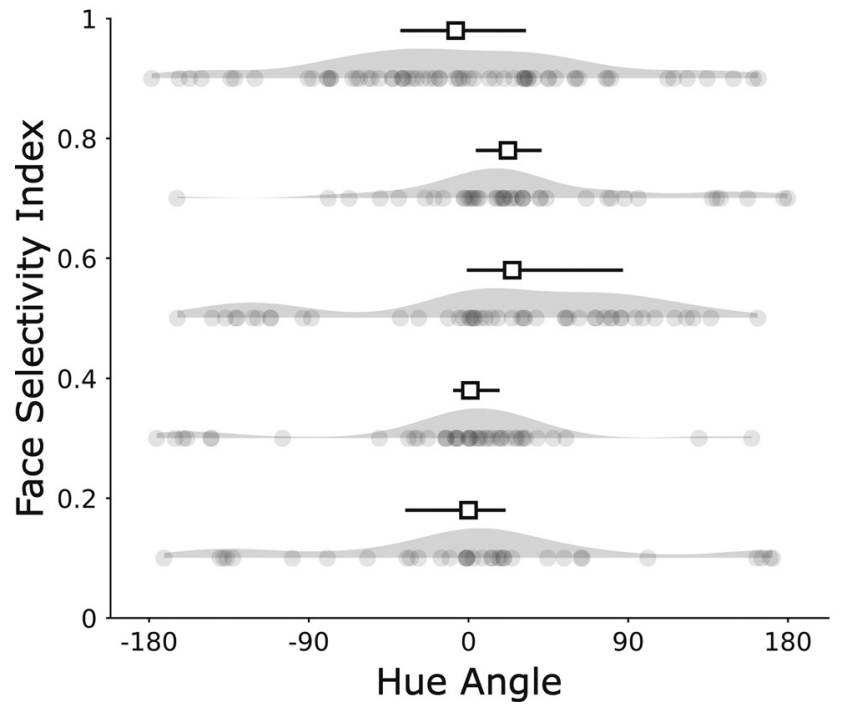

Figure 12. Hue preference by FSI. Each dot represents one cell in one bin of 0.2 . Median and $95 \%$ confidence intervals on the median for each bin are represented above the kernel density estimate of the distribution. The mean FSI across all 234 cells is 0.55 .

enhance the face-discrimination computations of the neurons. On average, faces have a warmer coloring than backgrounds (Rosenthal et al., 2018). It is plausible that the color responses would increase the firing rates of face-selective neurons when a face is encountered. Such modulation would presumably promote the role of these neurons in face recognition. According to this interpretation, the modulation by color of face-selective cells is analogous to the modulation of neural activity manifest when a subject engages in an attentional task (Wurtz and Mohler, 1976; Treue, 2001; Maunsell, 2015).

Is the color tuning we describe specific for faces? Quantitative analysis of the color statistics of those parts of scenes that we label, shows that objects tend to be systematically biased compared with backgrounds: objects, not just faces, tend to be distinguished from backgrounds along the u' direction of color space, which corresponds, roughly, to warm coloring (Gibson et al., 2017; Conway et al., 2020). Tuning to warm coloring may therefore facilitate the computations of many cells in IT, not just face-selective neurons. This hypothesis is supported by fMRI maps of the color tuning across IT, which show a band running along the posterior-anterior axis that is more strongly modulated by the likely colors of objects (Conway, 2018; Rosenthal et al., 2018). This band is centered on the face patches but is not restricted to them. Consistent with the fMRI results, we found that the non-face-selective cells, often found on the margins or just outside of face patches, also showed a weak bias for warm colors (Fig. 12). Moreover, others have reported that the optimal stimuli for IT cells are often of warm coloring (Ponce et al., 2019). We speculate that the modulation by color is likely not a specific property of face cells but may reflect a feature of IT that facilitates the computation by IT of object recognition generally.

Although most cells had a single peak in the color-tuning function, some face-selective neurons were best fit by two peaks, with maximum power to the second harmonic in the Fourier analysis (see example cell \#1; Fig. 4). The color selectivity of this subset of neurons, assessed as the phase angle of the second harmonic, was aligned with the S-cone axis in color space (Fig. 7). Is the tuning to the second harmonic meaningful? One possible concern could have been that these responses reflect a luminance artifact: estimates of equiluminance may not be accurate, especially for colors that modulate S-cones (Vos, 1978). We avoided this potential pitfall in the present work because the stimuli preserved the luminance contrast of the original images: the blackest and whitest regions in the colored versions of each image were the same as in the original image. Any luminance artifacts attributed to vagaries in the determination of equiluminance would be masked by preservation of the luminance contrast of the original image.

The response bias to colors along the $S$ axis was surprising; we think the results provide the first measurements of color tuning biases within extrastriate cortex that reflect the cardinal mechanisms. The cardinal mechanisms correspond to the color tuning of the cone-opponent cells that represent the first postreceptoral stage of color encoding and are reflected in the anatomy and physiology of the lateral geniculate nucleus (Derrington et al., 1984; Martin et al., 2001; Sun et al., 2006; Roy et al., 2009). The cardinal mechanisms are evident in behavioral work that is thought to isolate these subcortical contributions to color vision (Krauskopf et al., 1982; Eskew, 2009). The observation that cortical cells reflect the cardinal mechanisms is surprising because the distinct chromatic signatures associated with the cardinal mechanisms diffuse near the input layers to primary visual cortex (Tailby et al., 2008), and the organization of color undergoes progressively more uniform representation of color space through the visual-processing hierarchy (Bohon et al., 2016; Liu et al., 2020). The present results show that chromatic signatures corresponding to the cardinal mechanisms reemerge in extrastriate cortical circuits far along the putative visual-processing hierarchy, and they raise the possibility that the behavioral results reflecting the cardinal mechanisms may derive from responses not only of subcortical circuits, but also of extrastriate circuits.

\section{References}

Bohon KS, Hermann KL, Hansen T, Conway BR (2016) Representation of perceptual color space in macaque posterior inferior temporal cortex (the V4 complex). eNeuro 3:ENEURO.0039-16.2016.

Changizi MA, Zhang Q, Shimojo S (2006) Bare skin, blood and the evolution of primate colour vision. Biol Lett 2:217-221.

Chauhan T, Xiao K, Yates J, Wuerger S (2015) Estimating discrimination ellipsoids for skin images. J Vis 15:820.

Conway BR (2009) Color vision, cones, and color-coding in the cortex. Neuroscientist 15:274-290.

Conway BR (2001) Spatial structure of cone inputs to color cells in alert macaque primary visual cortex (V-1). J Neurosci 21:2768-2783.

Conway BR (2018) The organization and operation of inferior temporal cortex. Annu Rev Vis Sci 4:381-402.

Conway BR, Moeller S, Tsao DY (2007) Specialized color modules in macaque extrastriate cortex. Neuron 56:560-573.

Conway BR, Ratnasingam S, Jara-Ettinger J, Futrell R, Gibson E (2020) Communication efficiency of color naming across 
languages provides a new framework for the evolution of color terms. Cognition 195:104086.

Crichton S, Pichat J, Mackiewicz M, Tian G, Hurlbert AC (2012) Skin chromaticity gamuts for illumination recovery. In: Conference on color in graphics, imaging, and vision, pp 266-271. Springfield: Society for Imaging Science and Technology.

Derrington AM, Krauskopf J, Lennie P (1984) Chromatic mechanisms in lateral geniculate nucleus of macaque. J Physiol 357:241-265.

Edwards R, Xiao D, Keysers C, Földiák P, Perrett D (2003) Color sensitivity of cells responsive to complex stimuli in the temporal cortex. J Neurophysiol 90:1245-1256.

Eskew RT Jr (2009) Higher order color mechanisms: a critical review. Vision Res 49:2686-2704.

Freiwald WA, Tsao DY, Livingstone MS (2009) A face feature space in the macaque temporal lobe. Nat Neurosci 12:1187-1196.

Ganguli D, Simoncelli EP (2010) Implicit encoding of prior probabilities in optimal neural populations. Adv Neural Inf Process Syst 2010:658-666.

Gerald MS, Waitt C, Little AC (2009) Pregnancy coloration in macaques may act as a warning signal to reduce antagonism by conspecifics. Behav Processes 80:7-11.

Gibson E, Futrell R, Jara-Ettinger J, Mahowald K, Bergen L, Ratnasingam S, Gibson M, Piantadosi ST, Conway BR (2017) Color naming across languages reflects color use. Proc Natl Acad Sci USA 114:10785-10790. USA

Golz J, MacLeod DI (2002) Influence of scene statistics on colour constancy. Nature 415:637-640.

Hasantash M, Lafer-Sousa R, Afraz A, Conway BR (2019) Paradoxical impact of memory on color appearance of faces. Nat Commun 10:3010.

Horwitz GD, Hass CA (2012) Nonlinear analysis of macaque V1 color tuning reveals cardinal directions for cortical color processing. Nat Neurosci 15:913-919.

Johnson EN, Hawken MJ, Shapley R (2014) Cone inputs in macaque primary visual cortex. J Neurophysiol 91:2501-2514.

Kanwisher N, McDermott J, Chun MM (1997) The fusiform face area: a module in human extrastriate cortex specialized for face perception. J Neurosci 17:4302-4311.

Kemp R, Pike G, White P, Musselman A (1996) Perception and recognition of normal and negative faces: the role of shape from shading and pigmentation cues. Perception 25:37-52.

Komatsu H, Ideura Y (1993) Relationships between color, shape, and pattern selectivities of neurons in the inferior temporal cortex of the monkey. J Neurophysiol 70:677-694.

Krauskopf J, Williams DR, Heeley DW (1982) Cardinal directions of color space. Vision Res 22:1123-1131.

Kravitz DJ, Saleem KS, Baker Cl, Ungerleider LG, Mishkin M (2013) The ventral visual pathway: an expanded neural framework for the processing of object quality. Trends Cogn Sci 17:26-49.

Lafer-Sousa R, Conway BR (2013) Parallel, multi-stage processing of colors, faces and shapes in macaque inferior temporal cortex. Nat Neurosci 16:1870-1878.

Lafer-Sousa R, Conway BR, Kanwisher NG (2016) Color-biased regions of the ventral visual pathway lie between face- and place-selective regions in humans, as in macaques. J Neurosci 36:1682-1697.

Lefevre CE, Ewbank MP, Calder AJ, von dem Hagen E, Perrett DI (2013) It is all in the face: carotenoid skin coloration loses attractiveness outside the face. Biol Lett 9:20130633.

Leopold DA, Rhodes G (2010) A comparative view of face perception. J Comp Psychol 124:233-251.

Liu Y, Li M, Zhang X, Lu Y, Gong H, Yin J, Chen Z, Qian L, Yang Y, Andolina IM, Mcloughlin N, Tang S, Wang W, Shipp S (2020) Hierarchical representation for chromatic processing across macaque V1, V2, and V4. Neuron 108:538-550.e5.

Martin PR, Lee BB, White AJ, Solomon SG, Rüttiger L (2001) Chromatic sensitivity of ganglion cells in the peripheral primate retina. Nature 410:933-936.
Maunsell JHR (2015) Neuronal mechanisms of visual attention. Annu Rev Vis Sci 1:373-391.

Nakajima K, Minami T, Nakauchi S (2017) Interaction between facial expression and color. Sci Rep 7:41019.

Nestor A, Tarr MJ (2008) Gender recognition of human faces using color. Psychol Sci 19:1242-1246.

Ohayon S, Freiwald WA, Tsao DY (2012) What makes a cell face selective? The importance of contrast. Neuron 74:567-581.

Perrett DI, Rolls ET, Caan W (1982) Visual neurones responsive to faces in the monkey temporal cortex. Exp Brain Res 47:329-342.

Petersdorf M, Dubuc C, Georgiev AV, Winters S, Higham JP (2017) Is male rhesus macaque facial coloration under intrasexual selection? Behav Ecol 28:1472-1481.

Ponce CR, Xiao W, Schade PF, Hartmann TS, Kreiman G, Livingstone MS (2019) Evolving images for visual neurons using a deep generative network reveals coding principles and neuronal preferences. Cell 177:999-1009.e10.

Rosenthal I, Ratnasingam S, Haile T, Eastman S, Fuller-Deets J, Conway BR (2018) Color statistics of objects, and color tuning of object cortex in macaque monkey. J Vis 18:1.

Roy S, Jayakumar J, Martin PR, Dreher B, Saalmann YB, Hu D, Vidyasagar TR (2009) Segregation of short-wavelength-sensitive (S) cone signals in the macaque dorsal lateral geniculate nucleus. Eur J Neurosci 30:1517-1526.

Setchell JM, Wickings EJ (2005) Dominance, status signals and coloration in male mandrills (Mandrillus sphinx). Ethology 111:25-50.

Shevell SK, Kingdom FA (2008) Color in complex scenes. Annu Rev Psychol 59:143-166.

Simoncelli EP, Olshausen BA (2001) Natural image statistics and neural representation. Annu Rev Neurosci 24:1193-1216.

Sinha P, Balas BJ, Ostrovsky Y, Russell R (2006) Face recognition by humans: nineteen results all computer vision researchers should know about. Proc IEEE 94:1948-1962.

Stoughton CM, Lafer-Sousa R, Gagin G, Conway BR (2012) Psychophysical chromatic mechanisms in macaque monkey. $\mathrm{J}$ Neurosci 32:15216-15226.

Sun H, Smithson HE, Zaidi Q, Lee BB (2006) Specificity of cone inputs to macaque retinal ganglion cells. J Neurophysiol 95:837-849.

Tailby C, Solomon SG, Dhruv NT, Lennie P (2008) Habituation reveals fundamental chromatic mechanisms in striate cortex of macaque. J Neurosci 28:1131-1139.

Treue S (2001) Neural correlates of attention in primate visual cortex. Trends Neurosci 24:295-300.

Tsao DY, Freiwald WA, Tootell RB, Livingstone MS (2006) A cortical region consisting entirely of face-selective cells. Science 311:670-674.

Vos JJ (1978) Colorimetric and photometric properties of a $2^{\circ}$ fundamental observer. Color Res Appl 3:125-128.

Waitt C, Gerald MS, Little AC, Kraiselburd E (2006) Selective attention toward female secondary sexual color in male rhesus macaques. Am J Primatol 68:738-744.

Webster MA, MacLeod DI (2011) Visual adaptation and face perception. Philos Trans R Soc Lond B Biol Sci 366:1702-1725.

Wei XX, Stocker AA (2015) A Bayesian observer model constrained by efficient coding can explain 'anti-Bayesian' percepts. Nat Neurosci 18:1509-1517.

Wurtz RH, Mohler CW (1976) Enhancement of visual responses in monkey striate cortex and frontal eye fields. J Neurophysiol 39:766-772.

Xiao K, Yates JM, Zardawi F, Sueeprasan S, Liao N, Gill L, Li C, Wuerger S (2017) Characterising the variations in ethnic skin colours: a new calibrated data base for human skin. Skin Res Technol 23:21-29.

Yamashita JA, Hardy JL, De Valois KK, Webster MA (2005) Stimulus selectivity of figural aftereffects for faces. J Exp Psychol Hum Percept Perform 31:420-437. 